\title{
Statistical Optimization of FinFET Processor Architectures under PVT Variations Using Dual Device-Type Assignment
}

\author{
YE YU and NIRAJ K. JHA, Princeton University
}

\begin{abstract}
With semiconductor technology scaling to the $22 \mathrm{~nm}$ node and beyond, fin field-effect transistor (FinFET) has started replacing complementary metal-oxide semiconductor (CMOS), thanks to its superior control of short-channel effects and much lower leakage current. However, process, supply voltage, and temperature (PVT) variations across the integrated circuit (IC) become worse with technology scaling. Thus, to analyze timing, leakage power, and dynamic power under PVT variations, statistical analysis/optimization techniques are more suitable than traditional static timing/power analysis and optimization counterparts. In this article, we propose a statistical optimization framework using dual device-type assignment at the architecture level under PVT variations that takes spatial correlations into account and leverages circuit-level statistical analysis techniques. To the best of our knowledge, this is the first work to study statistical optimization at the system level under PVT variations. Simulation results show that leakage power yield and dynamic power yield at the mean value of the baseline can be improved by up to $44.2 \%$ and $21.2 \%$, respectively, with no loss in timing yield for a single-core processor and up to $43.0 \%$ and $50.0 \%$, respectively, without any loss in timing yield for an 8-core chip multiprocessor (CMP), at little area overhead. Under the same $(99.0 \%)$ power yield constraints, leakage power and dynamic power are reduced by up to $91.2 \%$ and $4.3 \%$, respectively, for a singlecore processor, and up to $44.6 \%$ and $12.5 \%$, respectively, for an 8-core CMP, with no loss in timing yield. We also show that optimizations performed without taking module-to-module and core-to-core spatial correlations into account overestimate yield, establishing the importance of taking such correlations into account.
\end{abstract}

CCS Concepts: • Computer systems organization $\rightarrow$ Architectures; $\bullet$ Hardware $\rightarrow$ VLSI system specification and constraints;

Additional Key Words and Phrases: Chip multiprocessor, FinFET, PVT variations, statistical analysis, statistical optimization

\section{ACM Reference format:}

Ye Yu and Niraj K. Jha. 2017. Statistical Optimization of FinFET Processor Architectures under PVT Variations Using Dual Device-Type Assignment. F. Emerg. Technol. Comput. Syst. 14, 1, Article 3 (September 2017), 25 pages.

https://doi.org/10.1145/3110714

\section{INTRODUCTION}

Traditional complementary metal-oxide semiconductor (CMOS) technology is facing considerable challenges in continued scaling beyond the $22 \mathrm{~nm}$ technology node, such as short-

This work was supported by the National Science Foundation under Grant No. CCF-1318603.

Authors' addresses: Y. Yu and N. K. Jha, Electrical Engineering Department, Princeton University, Princeton, NJ 08544; email: \{yeyu, jha\}@princeton.edu.

Permission to make digital or hard copies of part or all of this work for personal or classroom use is granted without fee provided that copies are not made or distributed for profit or commercial advantage and that copies show this notice on the first page or initial screen of a display along with the full citation. Copyrights for components of this work owned by others than ACM must be honored. Abstracting with credit is permitted. To copy otherwise, to republish, to post on servers, to redistribute to lists, or to use any component of this work in other works requires prior specific permission and/or a fee. Permissions may be requested from Publications Dept., ACM, Inc., 2 Penn Plaza, Suite 701, New York, NY 10121-0701 USA, fax + 1 (212) 869-0481, or permissions@acm.org.

(c) 2017 ACM 1550-4832/2017/09-ART3 $\$ 15.00$

https://doi.org/10.1145/3110714

ACM Journal on Emerging Technologies in Computing Systems, Vol. 14, No. 1, Article 3. Pub. date: September 2017. 
channel effects and intolerable leakage currents. Fin field-effect transistors (FinFETs) have emerged as a promising substitute in this regime because of their better control of the channel and immunity from random dopant fluctuations due to the use of a lightly doped channel. This enables FinFETs to deliver both low leakage and high performance. However, FinFETs still suffer from process, supply voltage, and temperature (PVT) variations, which become even more severe at the $22 \mathrm{~nm}$ node and beyond. Deterministic analysis, which estimates best-case, nominal, or worst-case power and delay, has been widely used in very large-scale integration (VLSI) design. However, it loses accuracy in analyzing circuits and architectures under PVT variations: best-case (worst-case) analysis significantly underestimates (overestimates) the impact of underlying variations, while analyzing circuits at nominal values of various circuit parameters completely ignores these variations. Hence, statistical analysis of power and delay has become necessary. It treats circuit parameters as random variables. Process variations are modeled as distributions of device parameters around their nominal values. The voltage difference across the integrated circuit (IC) is also modeled as a distribution around the nominal value. Variations have spatial correlations, which are of two types: die-to-die and within-die. Die-to-die variations affect devices across the die in a similar fashion. Under within-die variations, devices in the same neighborhood exhibit greater spatial correlation. Device variations, in turn, lead to variations in leakage power, dynamic power, and timing at the circuit and architecture levels. The corresponding IC yield can be estimated from the distributions of these parameters and specified target values for them.

Statistical analysis has been explored at various levels: logic circuit, cache, network-on-chip (NoC), pipeline, processor, and 3D IC (Yang and Jha 2014; Lee and Jha 2014; Mosaffa et al. 2016; Tang et al. 2014; Garg and Marculescu 2009a; Kim et al. 2015). It yields distributions of leakage power, dynamic power, and delay. In Yang and Jha (2014), variations in various FinFET parameter values as well as voltage and temperature variations were targeted. Various PVT variation models were derived: resistance/capacitance, temperature, dynamic power, leakage power, gate delay, output slope, and interconnect delay. The multi-level quad-tree model was used to account for spatial correlation. In Lee and Jha (2014), the impact of PVT variations on the cache and NoC was evaluated through macromodels at different levels: static random-access memory (SRAM) cell, subarray, cache bank, interconnect, NoC router, and so on. A statistical model for asynchronous pipelines based on Generalized Stochastic Petri Net in the presence of process variations was presented in Mosaffa et al. (2016). McPAT-PVT, which is a statistical delay and power modeling framework for FinFET-based processors, was presented in Tang et al. (2014). It contains delay/power macromodels at multiple levels of the processor module hierarchy. In Garg and Marculescu (2009a), the impact of process variations on the critical path delay of fully-synchronous 3D ICs was explored. Stochastic lower bound and upper bound were used for yield estimation. An approximation method for predicting the circuit delay of statistical static timing analysis through deterministic static timing analysis was presented in Kim et al. (2015), where the upper bound of the circuit delay was derived using a deterministic gate delay model.

The next step is to use statistical analysis as the basis for statistical optimization. Logic-level statistical optimization was explored using dual threshold voltage $\left(V_{t h}\right)$ assignment, gate sizing, and genetic algorithm in Hwang et al. (2013), Beece et al. (2014), Conos et al. (2013), Raji et al. (2011), and Tang and Jha (2016). A statistical optimization flow for yield-aware SRAM cell sizing and voltage selection under variability was presented in Singh et al. (2014), given the availability of post-silicon voltage tuning. A variability-aware 3D integration strategy for multiple clock-domain 3D systems was explored in Garg and Marculescu (2009b). It targets die-to-die variations, through die-level post-fabrication assembly.

It was shown that leakage power can be reduced by $25.2 \%$ using a statistical leakage minimization method, based on a gate change metric (Hwang et al. 2013). Power minimization in SRAM 
was formulated as a two-stage stochastic optimization problem (Singh et al. 2014), in which the first stage solves the gate-sizing problem and selects possible voltage levels, and the second stage assigns each partition to an optimal voltage after manufacturing. Dynamic programming was used to minimize power while meeting yield constraints on read, write, and static noise margins. It enables designs to be on average $8 \%$ and up to $17 \%$ more power-efficient than designs in which voltages are selected uniformly. It was reported that total power can be improved by up to $4.4 \times$ (on an average by $3.3 \times$ ) for the same timing and power yield constraints using a process variationaware near- $V_{t h}$ gate-sizing approach (Conos et al. 2013). Logic gates on the critical path are upsized to improve timing yield, while those on off-critical paths are downsized to reduce leakage power. In Raji et al. (2011), a statistical dual- $V_{t h}$ assignment of asynchronous circuits was proposed, which considers both the variability in performance and leakage power consumption of a circuit. It reduces leakage power mean and variance values by $40.5 \%$ and $54.4 \%$, respectively, with the help of a genetic algorithm. These works show the significant potential of statistical optimization. However, they are only applicable at the circuit level. A system-level statistical optimization method is still needed so that the whole IC can be optimized, and a high global yield improvement can be achieved based on local yield optimization and tradeoffs among lower-level components.

In this article, we make the following contributions:

(1) We propose a system-level statistical optimization framework, which makes use of lowerlevel statistical analysis, at the logic, cache, $\mathrm{NoC}$, pipeline, and processor levels in a bottom-up fashion. This framework takes important tradeoffs across different architectural modules into account and improves yield from a global perspective. To the best of our knowledge, this is the first study on statistical optimization at the system level under PVT variations.

(2) We employ the multi-level quad-tree model to calculate spatial correlations at all levels: transistor, functional unit, module, and core. We show the comparison of optimization results with and without module- and core-level spatial correlations taken into account, to establish the importance of taking them into account at all levels.

(3) We perform statistical optimization through a dual device-type assignment, where asymmetric-workfunction shorted-gate (ASG) FinFETs are used to improve leakage power yield and shorted-gate (SG) FinFETs are used for high performance. ASG FinFETs have very promising short-channel characteristics and have two orders of magnitude lower $I_{O F F}$ compared to that of SG FinFETs, with $I_{O N}$ only somewhat lower than that of SG FinFETs. Thus, by using dual device-type assignment, we can achieve dramatically more leakage power reduction compared to the traditional dual- $V_{t h}$ assignment used in CMOS circuit optimization. We show that ASG FinFETs can play a promising role in highperformance low-power architecture design.

Our simulation results show that leakage power and dynamic power yields can be increased by up to $44.2 \%$ and $21.2 \%$, respectively, for a single-core processor, and up to $43.0 \%$ and $50.0 \%$, respectively, for an 8-core chip multiprocessor (CMP), without any timing yield degradation. Leakage power and dynamic power are reduced at their $99.0 \%$ yield points by up to $91.2 \%$ and $4.3 \%$, respectively, for a single-core processor, and up to $44.6 \%$ and $12.5 \%$, respectively, for an 8-core CMP, with no timing yield loss.

The remainder of the article is organized as follows. Section 2 provides background information on FinFET structure and statistical analysis tools. Section 3 discusses our statistical optimization methodology. Section 4 presents simulation results. Section 5 discusses the insights derived from this work. Finally, Section 6 concludes the article. 


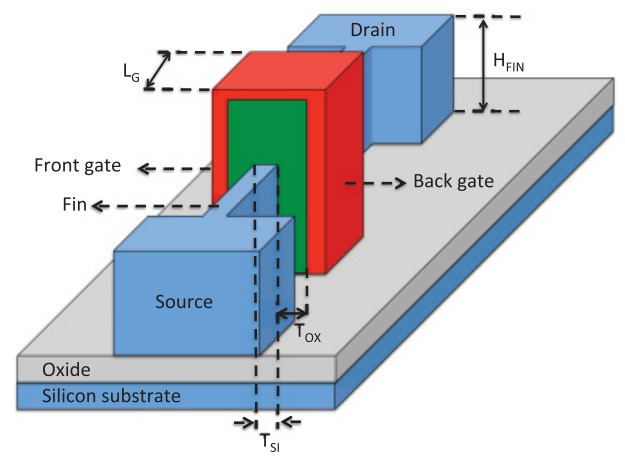

Fig. 1. 3D FinFET structure.

\section{BACKGROUND}

In this section, we provide background material necessary for understanding the rest of the article. We discuss the state-of-the-art of various FinFET types in Section 2.1 and describe our yield analysis methodology in Section 2.2. We describe the various simulators used in the framework in Sections 2.3-2.5.

\subsection{FinFET}

Figure 1 shows the 3D structure of a FinFET. Unlike a planar device, e.g., a MOSFET, a FinFET has a vertical channel shaped like a "fin" wrapped around by two gates: front gate and back gate. When the two gates are electrically connected at the top, the FinFET is called SG. When the top part is etched away, thus disconnecting the two gates, it is called independent-gate (IG). $L_{G}, T_{S I}, T_{O X}$, and $H_{F I N}$ refer to channel length, fin thickness, oxide thickness, and fin height, respectively. $\Phi_{N}$ and $\Phi_{P}$ represent the workfunctions of the $n$-type and $p$-type SG FinFETs, and $\Phi_{F}$ and $\Phi_{B}$ represent the workfunctions of the front and back gates of ASG FinFETs. The front and back gates have the same workfunctions in a symmetric SG FinFET, but different values in an ASG FinFET. An SG FinFET has around $30 \%$ higher on-current $\left(I_{O N}\right)$, but also two orders of magnitude higher off-current $\left(I_{O F F}\right)$ relative to an ASG FinFET. An IG FinFET has both lower $I_{O N}$ and an order of magnitude higher $I_{O F F}$ relative to an ASG FinFET. Thus, an IG FinFET is not useful for implementing highperformance circuits.

An ASG FinFET was first fabricated by IBM (Kedzierski et al. 2001) to realize low leakage power. Improvements were then made by various companies, such as Motorola and AMD (Mathew et al. 2003), and academia (Roy et al. 2006; Hussain et al. 2007). ASG FinFETs have a minimum fin thickness requirement due to process and design limitations. Its $I_{O N}$ is limited by the external parasitic resistance of the long fin extension region between the gate edge and probe pad. Additional masks are needed to realize an $\mathrm{N}+$ doped gate on one side of the fin and a $\mathrm{P}+$ doped gate on the other side. SG FinFETs are still predominantly used in the industry due to their lower fabrication cost. However, as FinFET scaling approaches sub-10nm technology nodes, the two orders of magnitude lower leakage power offered by ASG FinFETs become a very important consideration, making them promising for use in high-performance, ultra-low-power circuit design. Driven by this thinking, IBM has proposed a dual-workfunction integration process for stacked FinFETs in a recent patent (Cheng et al. 2016).

The dual device-type assignment we employ in this work can be compared to the dual- $V_{t h}$ assignment used in CMOS. Different optimization algorithms have been proposed for dual- $V_{t h}$ 
Table 1. 22-nm FinFET Parameter Values (Tang et al. 2014)

\begin{tabular}{cc}
\hline FinFET parameter & Value \\
\hline Fin height, $H_{F I N}(\mathrm{~nm})$ & 40 \\
Gate length, $L_{G}(\mathrm{~nm})$ & 20 \\
Fin thickness, $T_{S I}(\mathrm{~nm})$ & 10 \\
Oxide thickness, $T_{O X}(\mathrm{~nm})$ & 1 \\
Body doping, $N_{B}\left(\mathrm{~cm}^{-3}\right)$ & $10^{16}$ \\
Source/drain doping, $N_{S D}\left(\mathrm{~cm}^{-3}\right)$ & $10^{20}$ \\
Front/back gate thickness, $H_{F G} / H_{B G}(\mathrm{~nm})$ & 34 \\
Underlap near source/drain, $L_{U N}(\mathrm{~nm})$ & 5 \\
Fin pitch, $F_{P}(\mathrm{~nm})$ & 60 \\
Supply voltage, $V_{D D}(V)$ & 0.9 \\
Workfunctions (SG \& IG mode), $\Phi_{N} / \Phi_{P}(\mathrm{eV})$ & $4.4 / 4.8$ \\
Workfunctions (ASG mode), $\Phi_{F} / \Phi_{B}(\mathrm{eV})$ & $4.4 / 4.8$ \\
$3 \sigma / \mu$ & $10 \%$ \\
\hline
\end{tabular}

assignment, e.g., two-phase topology-based power minimization algorithm (Srivastava et al. 2004), and linear programming based $V_{t h}$ and gate-sizing joint assignment (Nguyen et al. 2003). These optimization algorithms can also be employed in the context of dual device-type assignment. However, dual device-type assignment can achieve dramatically more leakage power reduction compared to dual- $V_{t h}$, especially at sub-10nm nodes. To continue FinFET scaling to sub-10nm nodes, material and device architecture modifications are necessary for combating poor short-channel effects and new leakage mechanisms, such as direct source-to-drain tunneling. Various FinFET structures have been studied for targeting sub-10nm nodes: asymmetric underlap (Akkala et al. 2016), dual- $k$ spacer (Dutta et al. 2016), contact-all-around (Chou et al. 2016), and ASG (Bhoj and Jha 2014). In addition to the device structure, new materials have also been studied at sub-10nm nodes, e.g., use of $\mathrm{Ge}$ as a replacement of Si owing to its higher carrier mobility (Sinha et al. 2015). Ge has demonstrated higher SoC performance at the 10nm and 7nm nodes Bardon et al. (2014). A 3D technology computer-aided-design (TCAD)-based methodology has been proposed for parasitic capacitance extraction of FinFET devices at the 10nm node (Bhoj et al. 2013). To account for the emerging quantum effects at sub-10nm nodes, accurate and efficient device simulations become necessary. However, the traditional drift-diffusion and hydrodynamic (HD) device simulation models are no longer accurate in this regime. The quantum hydrodynamic (QHD) model becomes essential for device simulation. However, it suffers from poor convergence and high simulation times. A methodology for speeding up simulation and improving convergence has been proposed that captures the device states from the classical HD model and then feeds the results as an initial guess to QHD simulation, based on the Newton-Raphson method (Dai and Jha 2017). This method can be applied to sub-10nm FinFET cell library characterization, which can then be used in the architectural simulators targeted at these FinFET nodes.

Table 1 shows the 22-nm FinFET parameter values we use for simulation results in this article. The $L_{G}, T_{S I}$, and $T_{O X}$ values were obtained from ITRS (2013). The $H_{F I N}, N_{B}, N_{S D}, H_{F G}, H_{B G}, L_{U N}$, and $F_{P}$ values were selected based on data from the foundries (Guillorn et al. 2008; Wu et al. 2010; Yamashita et al. 2011). We consider variations in two groups of process parameters (Chaudhuri. and Jha 2014; Xiong and Bokor 2003; Choi et al. 2007; Mishra et al. 2010): structural parameters and workfunctions. Structural parameters include $L_{G}, T_{S I}$, and $T_{O X}$. Variations in workfunctions alter the $V_{t h}$, thus impacting both leakage current and delay. These process variations are set to $3 \sigma / \mu=10 \%$, as typically assumed in statistical analysis of such devices. 


\subsection{Yield Analysis Methodology}

Since the impact of PVT variations is becoming increasingly severe in the FinFET era, it is important to optimize IC yield. The yield of an IC is defined as the fraction of manufactured ICs that satisfy all the design requirements. Thus, the yield can be defined as the probability that the IC meets the requirement. This can be estimated by the cumulative distribution function (CDF) at the given constraint (Tang et al. 2014):

$$
\begin{gathered}
T Y(t)=P\left(t \leq T_{\text {clock_period }}\right)=\int_{0}^{T_{\text {clock_period }}} f(t) d t \\
P Y(p)=P\left(p \leq P_{\text {budget }}\right)=\int_{0}^{\text {Power budget }} f(p) d p
\end{gathered}
$$

where $T Y(\cdot)$ and $P Y(\cdot)$ represent timing yield and power yield, respectively. $P(\cdot)$ denotes probability, $f$ denotes the probability density function (PDF), $T_{\text {clock_period }}$ is the clock period, and $P_{\text {budget }}$ is the power budget of the component.

\subsection{Statistical Analysis of FinFET Logic Circuits under PVT Variations}

Although FinFETs do not suffer from random dopant fluctuations due to their lightly-doped channel, their electrical properties are still significantly impacted by PVT variations.

We use FinPrin (Yang and Jha 2014), which is a statistical power/delay analysis tool for FinFETbased logic circuits, in our evaluations. Based on a FinFET standard cell library (Bhoj and Jha 2013; Chaudhuri and Jha 2011; Chaudhuri. and Jha 2014), FinPrin obtains PVT variation-aware statistical models for circuit-level leakage power, dynamic power, and delay. The statistical delay model is based on statistical static timing analysis (Chang and Sapatnekar 2005b) and Horowitz's delay model (Horowitz 1983). The statistical leakage model uses the macromodels presented in Mishra et al. (2010). Spatial correlations are taken into account using a rectangular grid-based quadtree model (Agarwal et al. 2003). The distribution of random variables is obtained using statistical sum and max operations. The analytical formula for statistical max is borrowed from Clark (1961) and extended to multiple random variables using the method in Chang and Sapatnekar (2005b). When spatial correlation is taken into account, obtaining the statistical sum and max of a group of random variables becomes computationally expensive. Thus, principal component analysis is used to translate the correlated random variables to a different coordinate system where they are independent of each other.

\subsection{FinCANON}

FinCANON (Lee and Jha 2014) is a FinFET-based PVT variation-aware delay and power modeling framework for caches and NoCs, under spatial correlations. It incorporates voltage generators in the FinFET design library to model back-gate biasing of IG FinFETs. FinCANON is modular and scalable. Thus, models for new SRAM cells or router pipelines can be easily incorporated into it. It has been evaluated with both synthetic and real traffic obtained from the Princeton Application Repository for Shared-Memory Computers (PARSEC) benchmark suite (Bienia et al. 2008). It consists of two tools: CACTI-PVT for caches and ORION-PVT for NoCs, which are discussed next.

2.4.1 CACTI-PVT. CACTI-PVT is built atop CACTI (Wilton and Jouppi 1996), a widely used tool from HP Labs that models cache area, power, and access delay hierarchically. Logical effort is employed to optimize transistor sizes and delay. The circuit-level parameters it uses, such as input capacitance $\left(C_{I n}\right)$, output capacitance $\left(C_{O u t}\right)$, output resistance $\left(R_{O u t}\right)$, and leakage current $\left(I_{\text {Leak }}\right)$, are obtained from the FinFET design library. The leakage power of each cache component 
is modeled as a lognormal random variable, since the leakage power of a gate is modeled as an exponential function, as follows (Yang and Jha 2014):

$$
P_{\text {Leak }}=c \cdot e^{a_{0}+a_{1} L_{G}+a_{2} T_{S I}+a_{3} T_{O X}+a_{4} \Phi_{N}+a_{5} \Phi_{P}^{2}+a_{6} \Phi_{P}+a_{7} V_{D D}},
$$

where $c$ and $a_{0}-a_{7}$ are fitting coefficients. SRAM cell models are characterized using accurate TCAD simulations. Various SRAM cell types are supported: row-based back-gate biasing (RBGB) 6T (Joshi et al. 2007), pass-gate feedback (PGFB) 6T (Carlson et al. 2006), 4T (Guo et al. 2005), 8T (Kim et al. 2008), and ASG 6T cell (Bhoj and Jha 2014). Peripheral circuits implemented with various FinFET types (SG, IG, ASG) are supported. Its output includes a delay profile and a power profile. The delay profile includes the search delay of the tag array, the write/read delay of the data array, and the delay spread information. The power profile includes the write/read energy per access and the dynamic/leakage power breakdown of the entire cache.

2.4.2 ORION-PVT. ORION-PVT is built atop the ORION infrastructure (Wang et al. 2002), which targets bulk CMOS-based NoC simulation. The power consumption and delay of buffers, crossbar, arbiters, clock tree, and links are modeled using the FinFET design library under PVT variations, using network traffic statistics. It uses SRAM models from CACTI-PVT for this purpose.

\subsection{McPAT-PVT}

McPAT-PVT (Tang et al. 2014) is a statistical power/delay analysis framework for FinFET processors under PVT variations. It integrates the FinFET design library, FinPrin, and FinCANON, with its functional block macromodels embedded in an architecture-level macromodel hierarchy. It fetches the processor configuration from the system simulation tool, gem5 (Binkert et al. 2011), which specifies the operating temperature, supply voltage, technology, configurations of processor components, and the processor behavior profile, e.g., number of cache accesses and hit rate. It simulates processor power and delay in a bottom-up fashion: transistor-level PVT variations are taken into account in the FinFET design library and their impact propagated to functional block macromodels. Caches and NoCs are simulated using FinCANON. The outputs of FinCANON and functional block macromodels are then incorporated into the architecture-level macromodel hierarchy of the processor. Spatial correlations between transistors within functional units and modules are accounted for by using the multi-level quad-tree model. However, McPAT-PVT does not consider spatial correlations between modules or between cores.

\section{METHODOLOGY}

In this section, we first describe the simulation framework we employ for statistical optimization and various macromodels used in the processor hierarchy. We then discuss local optimization methods employed at the functional unit and module levels. These methods yield locally optimal candidates. Then, we describe our method for obtaining globally optimal solutions for the whole processor from the lower-level locally optimal candidates. Finally, we discuss how spatial correlations between modules and cores can be taken into account.

\subsection{Statistical Optimization Simulation Framework and Macromodel Hierarchy}

Figure 2 shows the simulation framework we use for statistical optimization. McPAT-PVT and FinCANON take processor configuration as input, which specifies operating temperature, supply voltage, technology node, and configurations of processor components, e.g., number of cores, cache size, and pipeline type. The processor behavior profile, e.g., number of instructions, cache access amount, and hit rate, is also included in the processor configuration for dynamic power and delay simulations. It is obtained from the gem5 full system simulator (Binkert et al. 2011). 


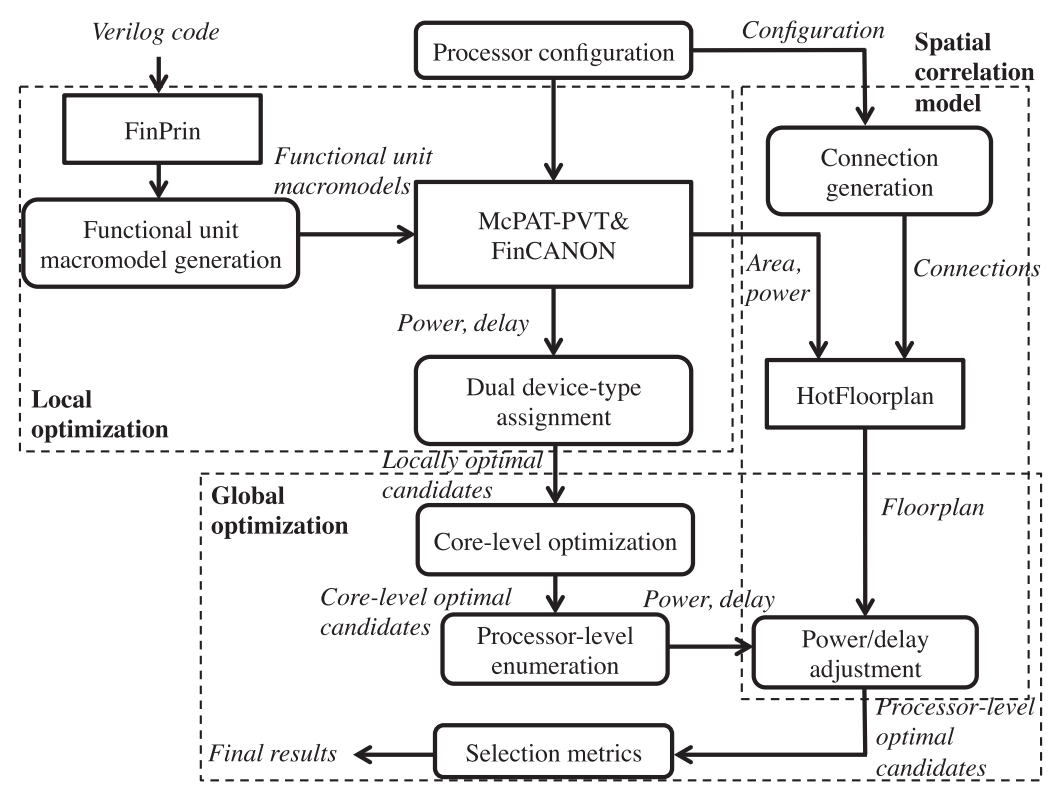

Fig. 2. Simulation flow for statistical optimization.

McPAT-PVT contains an architectural macromodel hierarchy, where the lowest-level macromodels (functional unit macromodels) are generated from FinPrin, and the power and timing distributions of the higher-level macromodels are computed from those of the lower-level ones using statistical max and sum operations. Power distributions are computed using the statistical sum of the components' power distributions. Timing distributions are computed using both statistical sum and max, depending on whether the components are connected in series or parallel. Dual device-type assignment is performed at various levels of the processor hierarchy in McPAT-PVT: each submodule inside the processor is implemented with SG, ASG, and SG/ASG hybrid libraries, and the locally optimal candidates are selected by comparing the leakage power and delay values of different device-type implementations at a certain yield. These locally optimal candidates are then used in core-level and processor-level optimizations, where full-chip simulation is performed using these candidates, through an exhaustive consideration of all combinations. The simulation results are then updated using the spatial correlation model, in which module-to-module and coreto-core spatial correlations are taken into account. Then, the delay and power values of all the processor-level results are compared and globally attractive solutions are selected using some filtering conditions. The final results contain the device-type assignment of each submodule in the processor and the corresponding delay/power distributions.

Figure 3 shows the block diagram of the McPAT-PVT processor model (Tang et al. 2014) used in this article. It includes gate-level macromodels defined in the FinFET design library (Lee and Jha 2014), circuit-level macromodels, and architecture-level macromodels in a hierarchical manner. The FinFET design library provides macromodels of FinFET logic gates and memory cells, targeted at capacitance, resistance, leakage, area, and delay. Leakage power, dynamic power, and delay are estimated at the circuit level using the power variation, delay variation, and temperature variation models. These variation values are then used in cache and $\mathrm{NoC}$ macromodels provided in FinCANON (Lee and Jha 2014), and functional unit macromodels generated using FinPrin (Yang and Jha 2014). Macromodels of higher-level processor components in the architecture hierarchy are built based on these circuit-level macromodels. Finally, delay and power variation profiles as 


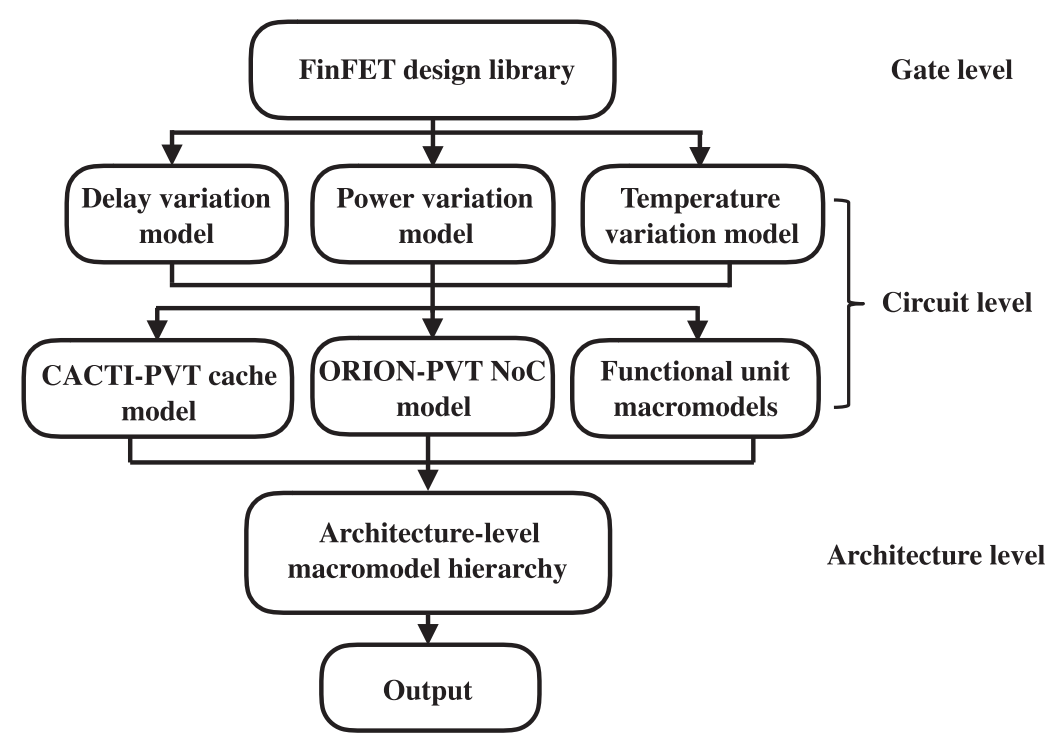

Fig. 3. Block diagram of the McPAT-PVT processor model (Tang et al. 2014) @IEEE.

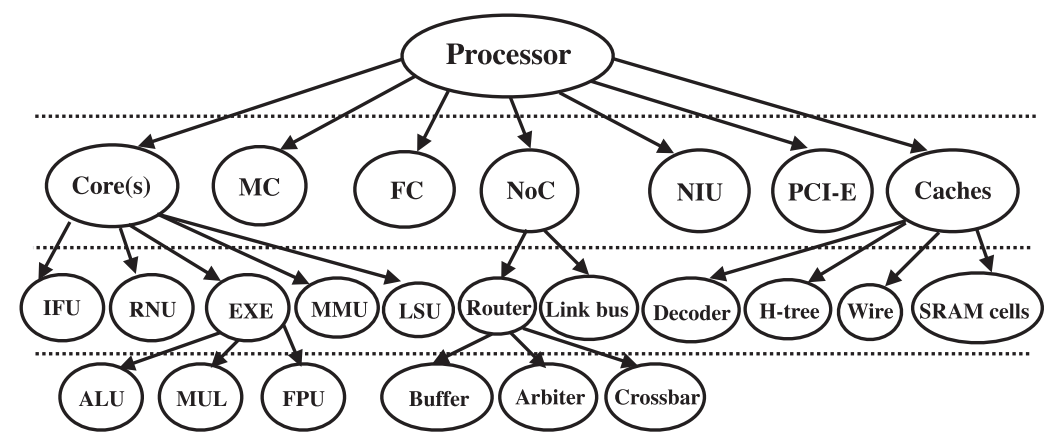

Fig. 4. The processor macromodel hierarchy in McPAT-PVT (Tang et al. 2014) OIEEE.

well as an area estimate are generated from the processor macromodel, the topmost level in the architecture-level macromodel hierarchy of McPAT-PVT.

Figure 4 shows the processor model hierarchy inherited from McPAT-PVT. There are four different levels in this hierarchy. At each level, macromodels are used to characterize each component of the processor. These macromodels not only estimate the nominal delay and power values, but also their variations. The topmost level defines the processor macromodel. The second level consists of macromodels of the main components in the processor, e.g., cores, L2/L3 caches, memory controller $(\mathrm{MC})$, flash controller (FC), NoC, network interface unit (NIU), and peripheral component interconnect express (PCI-E) controller. More components can easily be added to the processor model hierarchy. The third level contains macromodels for the major components of cores, caches, and NoCs. It includes macromodels for the instruction fetch unit (IFU), renaming unit (RNU), execution unit (EXE), memory management unit (MMU), and load-store unit (LSU) under cores. Router and link bus are defined under NoC. Decoder, H-tree, wire, and SRAM cell macromodels are defined under caches at this level. Macromodels at the third level can be constituted from macromodels of smaller components at the lowest level. For example, EXE contains the arithmetic logic unit (ALU), 
multiplier (MUL), floating-point unit (FPU), and so on. The router consists of the buffer, arbiter, and crossbar. These macromodels in the hierarchy are generated either from register-transfer level (RTL) simulations or from FinCANON (Lee and Jha 2014). The L2 cache can be modeled as a shared or private cache structure in each core.

Unlike the traditional paradigm in architectural simulators that rely on scaling of results from a previous technology node, we characterized the FinFET standard cell and SRAM cell libraries through accurate device simulations (Yang and Jha 2014) using a TCAD tool: Sentaurus TCAD (Synopsys 2016b). The FinFET device dimensions used in the library are based on those used in the industry (Guillorn et al. 2008; Wu et al. 2010; Yamashita et al. 2011). The following physics models were used in device simulation: effective intrinsic density, mobility, Fermi, hydrodynamic, and recombination. Effective intrinsic density is a model that takes into account the silicon bandgap narrowing effect in highly doped regions. The mobility physics model includes the effect of electron or hole mobility degradation due to high doping, carrier velocity saturation in high-field regions, and at semiconductor-insulator interfaces. The Fermi model sets the driving force for carrier velocity saturation due to high electric field to the gradient of the quasi-Fermi potential. The hydrodynamic model is used to solve the carrier temperature and heat flow equations, which are essential components of deep-submicron device simulations. Recombination models account for the doping- and temperature-dependent Shockley-Read-Hall (SRH) recombination. These device simulations took several CPU months of effort.

The accuracy bubbles up from the device level to the circuit level since the FinFET design library (standard and SRAM cells) is used in FinPrin to analyze PVT variations and generate functional unit macromodels at the circuit level. FinPrin has been validated against accurate quasi MonteCarlo simulations, incurring test error less than 5\% (Yang and Jha 2014). As shown in Figure 3, the gate-level accuracy of the FinFET design library also propagates to the circuit level through the delay variation, power variation, and temperature variation models defined in CACTI-PVT and ORION-PVT, and to the architecture level through the macromodel hierarchy defined in McPATPVT. In order to validate the architectural macromodels in the McPAT-PVT hierarchy, we compare the probability of timing error due to PVT variations of an instruction cache and an FPU in an Alpha processor obtained from McPAT-PVT with the estimation from VARIUS (Sarangi et al. 2008), a PVT-aware microarchitecture-level model that has been validated against empirical error rate data. A good match is obtained, as shown in Figure 5. HotFloorplan has been validated as part of HotSpot against two test chips (a thermal test chip from MicReD and an FPGA) (Zhang et al. 2015).

\subsection{Local Optimization of Functional Units and Modules}

Since dynamic power is not very sensitive to device variations, but only to operating voltage and frequency, we only focus on leakage power and timing yield improvements during local optimization. Let us first consider macromodel generation for functional units present in an execution unit, i.e., ALU, multiplier, and floating-point unit at the lowest level in Figure 4. The macromodels of these three components are obtained using Synopsys Design Compiler (Synopsys 2016a) and FinPrin (Yang and Jha 2014), as shown in Figure 6 (Tang et al. 2014). Their RTL Verilog description is provided as input to Synopsys Design Compiler with two different standard cell libraries: SG and ASG. Each functional unit is synthesized several times, targeting different frequencies of operation. Synthesized gate-level netlists are generated by Design Compiler under various constraints. FinPrin then takes these netlists as input for PVT variation analysis under different temperatures. FinPrin simulation results for two target frequencies are shown in Table 2. Based on these results, we choose the ASG FinFET implementation with a $1 \mathrm{GHz}$ target frequency as the starting point for ALU macromodel generation, through regression and parameter extraction, since it meets the 


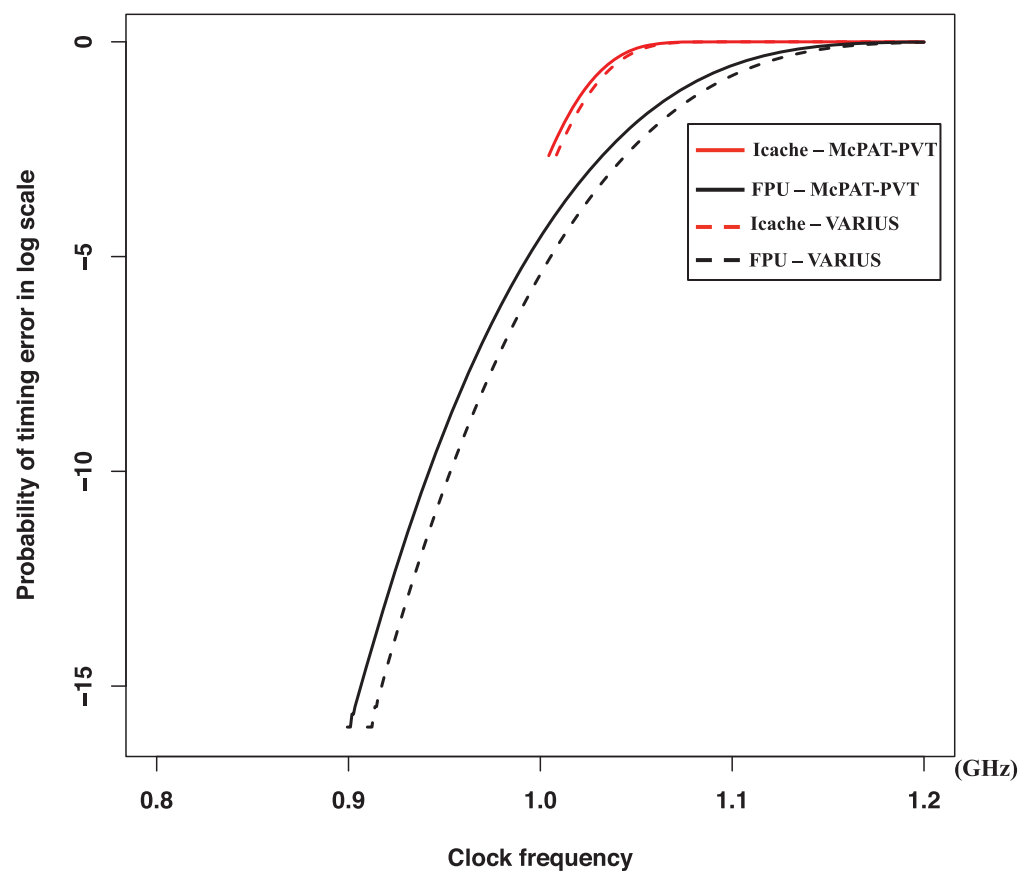

Fig. 5. Validation of McPAT-PVT for logic and memory models.

timing requirement with least dynamic/leakage power. We choose both SG and ASG FinFET implementations with a $1 \mathrm{GHz}$ target frequency as the starting points for floating-point unit and multiplier macromodel generation because SG implementations satisfy the timing requirement while the ASG counterparts have significantly lower dynamic/leakage power, though they violate the timing requirement slightly. This can be resolved by upsizing the gates in the circuit. The gatesizing assignment is changed iteratively from the starting configuration of each functional unit, followed by a FinPrin simulation, from which a power/delay pair is obtained in each iteration. The functional unit macromodel is then generated through regression and parameter extraction based on these power/delay pairs. A functional unit macromodel is realized as follows (Tang et al. 2014):

$$
G\left(T_{o p}, F_{o p}\right)=G_{\text {base }} \cdot e^{G_{t e m p}\left(T_{o p}\right)} \cdot G_{f r e q}\left(F_{o p}\right)
$$

The functional unit macromodel takes the effect of both temperature and frequency into account: $T_{o p}$ is the operation temperature, $F_{o p}$ denotes the operation frequency, and $G_{b a s e}$ is a constant associated with different functional units. $G_{t e m p}$ and $G_{\text {freq }}$ are polynomials that determine the temperature and frequency variation trends, respectively. All of them are obtained through regression that minimizes residual error. The macromodels at upper levels of the processor hierarchy in McPAT-PVT are built on top of these functional unit macromodels.

We simulate L1/L2 caches (of size 64KB/8MB) using CACTI-PVT (Lee and Jha 2014). We do not consider 4T and 8T SRAM cells defined in CACTI-PVT due to their low noise margin or high area overhead, respectively. Hence, we simulate three SRAM cells: RBGB 6T, PGFB 6T, and ASG 6T, with three peripheral circuit implementation styles: SG, ASG, and hybrid (Chen and Jha 2014). In the hybrid peripheral circuit implementation, we replace three SG FinFET buffers inside the decoder with ASG FinFET ones. This reduces leakage power to up to $15.1 \%$ of the SG baseline, while the delay only goes up by $0.4 \%$ relative to the SG baseline (Chen and Jha 2014). We also implement repeaters in the data H-tree using ASG FinFETs in the hybrid peripheral circuitry, and the rest of 


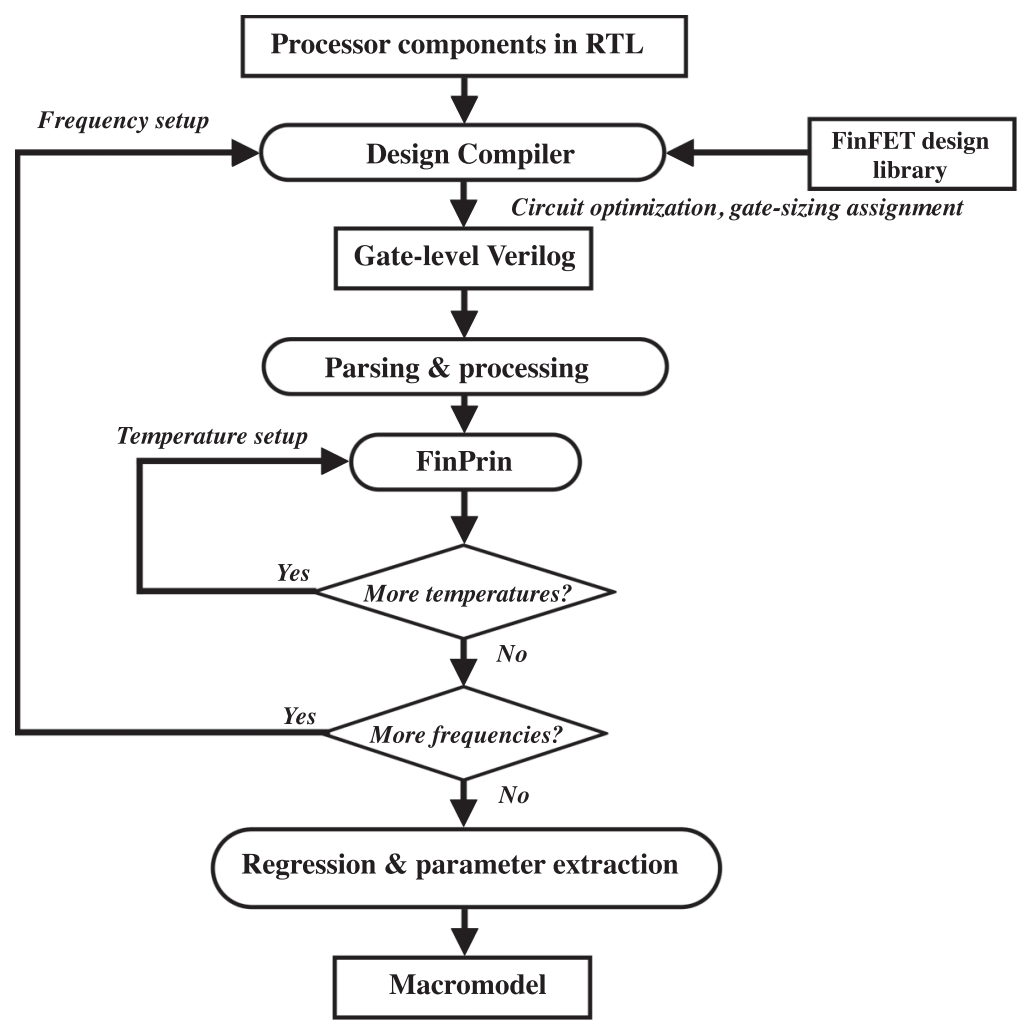

Fig. 6. Functional unit macromodel generation flow (Tang et al. 2014) @IEEE.

the cache is built using SG FinFETs. The hybrid H-tree consumes 95\% less leakage power while incurring 8.3\% delay overhead, compared to the SG baseline (Chen and Jha 2014).

For L1 cache simulations, we use a 64KB, 4-way least recently used (LRU), 64B block, four uniform cache access (UCA) bank, and 64-bit address structure. We explore the nine SRAM cell/peripheral circuit combinations mentioned above. We compare leakage power and timing at their $99.0 \%$ yield point (at which value, the yield becomes 99.0\%) in Figure 7. We choose the 99.0\% yield point for comparison as it lies between the two-sigma range (95.0\%) and three-sigma range (99.7\%) of normal distributions that are often employed. However, our method is independent of this choice. Configurations of each combination are shown in the legend alongside, where PGFB, RBGB, and ASG 6T denote the SRAM cell type, while SG, ASG, and hybrid denote the peripheral circuit implementation. To achieve a higher yield, a smaller $99.0 \%$ yield point would be preferred. We use an 8MB, 8-way LRU, 64B block, four UCA bank, 64-bit address cache structure for L2 cache simulation. We show the leakage power/timing comparisons at $99.0 \%$ yield point in Figure 8 . In both cases, use of the ASG 6T SRAM cell along with either SG or ASG or hybrid SG/ASG implementation of the cache peripheral circuitry leads to non-dominated solutions (i.e., no other combination dominates them with smaller values in both leakage power and timing). Thus, these three combinations are chosen as cache candidates for global optimization.

We simulate the components of processor cores using the macromodels available from the third level of the McPAT-PVT macromodel hierarchy (Figure 4). We simulate each component (instruction fetch unit, renaming unit, load-store unit, memory management unit, execution unit, etc.) 
Table 2. FinPrin Simulation Results

\begin{tabular}{|c|c|c|c|c|}
\hline Functional Unit & FPU & FPU & FPU & FPU \\
\hline Device Mode & SG & SG & ASG & ASG \\
\hline Target Frequency $(\mathrm{GHz})$ & 1 & 2 & 1 & 2 \\
\hline Area $\left(\mathrm{um}^{2}\right)$ & $51,103.1$ & $55,084.5$ & $58,824.3$ & $63,028.1$ \\
\hline Delay Mean $(n s)$ & 0.964 & 0.714 & 1.033 & 0.746 \\
\hline Delay Sigma $(n s)$ & 0.032 & 0.027 & 0.023 & 0.016 \\
\hline Dynamic Power Mean $(\mu W)$ & $6,512.1$ & $15,487.7$ & $4,734.6$ & $11,364.3$ \\
\hline Dynamic Power Sigma $(\mu W)$ & 515.0 & $1,218.7$ & 421.1 & 991.0 \\
\hline Leakage Power Mean $(\mu W)$ & $4,544.5$ & $5,355.1$ & 68.0 & 81.1 \\
\hline Leakage Power Sigma $(\mu W)$ & $1,240.5$ & $1,455.0$ & 6.7 & 7.6 \\
\hline
\end{tabular}

\begin{tabular}{|c|c|c|c|c|}
\hline Functional Unit & ALU & ALU & ALU & ALU \\
\hline Device Mode & SG & SG & ASG & ASG \\
\hline Target Frequency $(\mathrm{GHz})$ & 1 & 2 & 1 & 2 \\
\hline Area $\left(u m^{2}\right)$ & $4,382.7$ & $4,528.6$ & $4,878.2$ & $5,580.0$ \\
\hline Delay Mean $(n s)$ & 0.770 & 0.495 & 0.945 & 0.583 \\
\hline Delay Sigma $(n s)$ & 0.033 & 0.021 & 0.024 & 0.013 \\
\hline Dynamic Power Mean $(\mu W)$ & 256.2 & 593.0 & 194.9 & 452.4 \\
\hline Dynamic Power Sigma $(\mu W)$ & 20.0 & 45.7 & 16.6 & 38.1 \\
\hline Leakage Power Mean $(\mu W)$ & 176.6 & 208.9 & 2.6 & 3.1 \\
\hline Leakage Power Sigma $(\mu W)$ & 49.1 & 57.4 & 0.3 & 0.3 \\
\hline
\end{tabular}

\begin{tabular}{|c|c|c|c|c|}
\hline Functional Unit & MUL & MUL & MUL & MUL \\
\hline Device Mode & SG & SG & ASG & ASG \\
\hline Target Frequency $(\mathrm{GHz})$ & 1 & 2 & 1 & 2 \\
\hline Area $\left(\mathrm{um}^{2}\right)$ & $13,479.4$ & $16,656.5$ & $20,754.9$ & $24,333.6$ \\
\hline Delay Mean $(n s)$ & 0.931 & 0.677 & 1.041 & 0.713 \\
\hline Delay Sigma $(n s)$ & 0.035 & 0.025 & 0.024 & 0.013 \\
\hline Dynamic Power Mean $(\mu \mathrm{W})$ & $2,351.2$ & $5,805.2$ & $1,735.7$ & $4,295.8$ \\
\hline Dynamic Power Sigma $(\mu \mathrm{W})$ & 184.3 & 453.9 & 149.4 & 369.6 \\
\hline Leakage Power Mean $(\mu \mathrm{W})$ & $1,572.9$ & $1,959.3$ & 24.5 & 30.3 \\
\hline Leakage Power Sigma $(\mu \mathrm{W})$ & 422.7 & 528.4 & 2.1 & 2.6 \\
\hline
\end{tabular}

using three different libraries: SG, ASG, and SG logic with hybrid peripheral circuit SRAM (if no SRAM is in the component, then it reduces to the SG library). The SRAM cell type is fixed to ASG $6 \mathrm{~T}$ for all the three libraries based on the L1/L2 cache simulations discussed above. Figure 9(a)-(e) show the leakage power and timing of each component with these libraries at its $99.0 \%$ yield points. We select non-dominated candidates for each component: SG and ASG for instruction fetch unit and memory management unit; ASG and SG+hybrid SRAM for renaming unit, load-store unit, and execution unit. We use these candidates for further global optimization. We model various sub-components, e.g., register file and instruction scheduler, in the same manner.

We simulate NoC routers using the macromodels available from ORION-PVT, again using the three SG, ASG, and SG+hybrid SRAM libraries. We compare leakage power and timing at the $99.0 \%$ yield point in Figure 9(f). We choose the non-dominated ASG and SG+hybrid SRAM configurations as candidates for further optimization. 


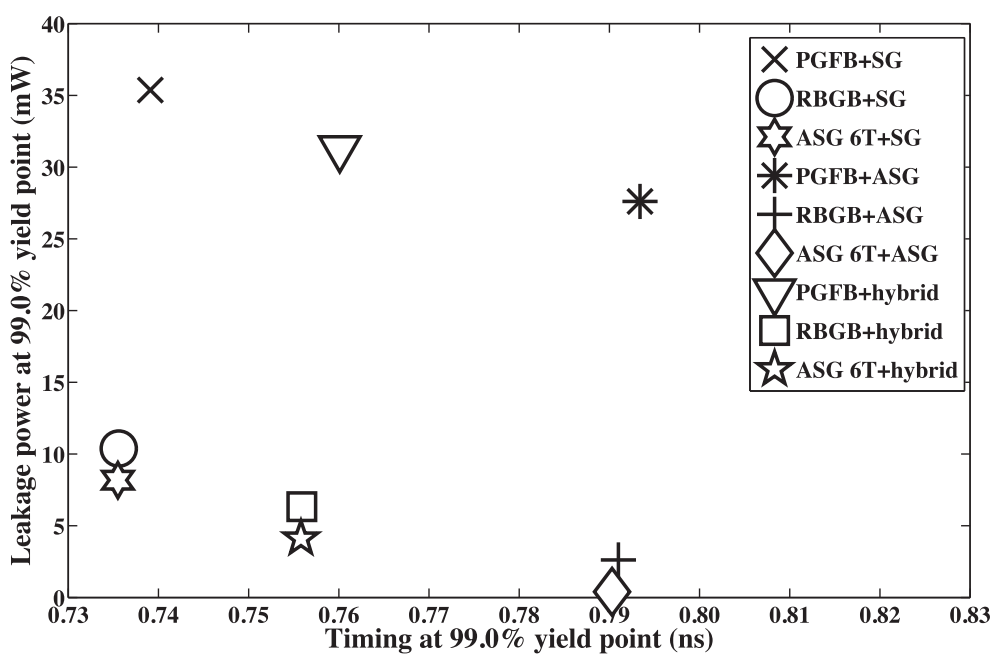

Fig. 7. Leakage power and timing comparisons of different $\mathrm{L} 1 \mathrm{cell} /$ peripheral circuit combinations at $99.0 \%$ yield points. PGFB, RBGB, and ASG 6T denote the SRAM cell type, while SG, ASG, and hybrid denote the peripheral circuit implementation.

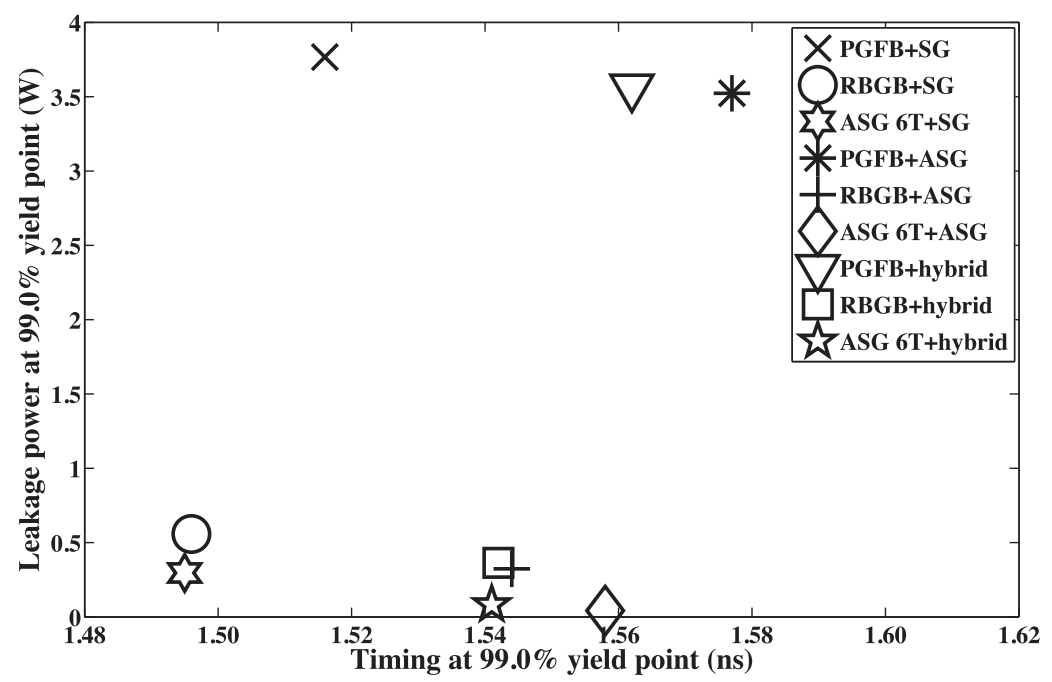

Fig. 8. Leakage power and timing comparisons of different L2 cell/peripheral circuit combinations at $99.0 \%$ yield points. PGFB, RBGB, and ASG 6T denote the SRAM cell type, while SG, ASG, and hybrid denote the peripheral circuit implementation.

\subsection{Global Optimization of the Processor}

We use the topmost macromodel in the McPAT-PVT hierarchy for global optimization of the processor. We simulate each component or sub-component inside the processor using the non-dominated (locally optimal) candidates derived in the previous step. We first enumerate all combinations of all candidates in processor cores, i.e., the core is simulated multiple times with one module configuration changed each time based on local optimization results. We obtain leakage 


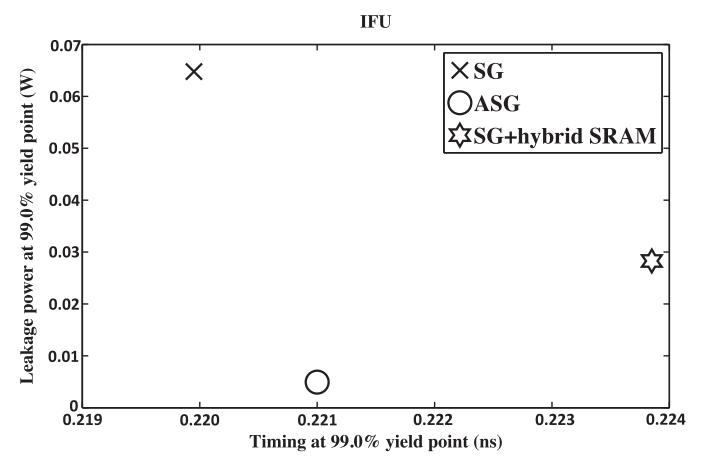

(a) Instruction fetch unit

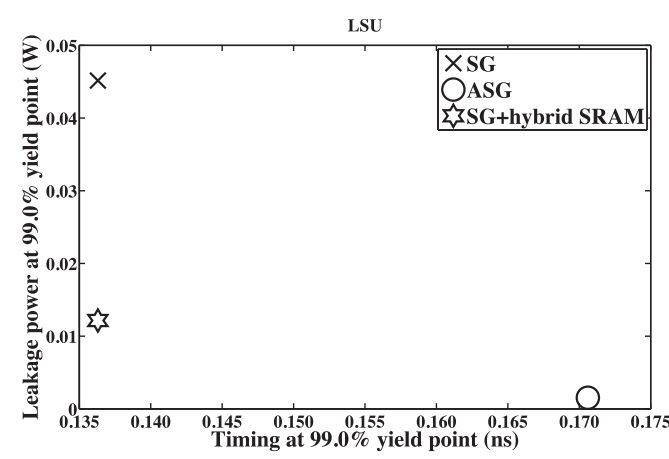

(c) Load-store unit

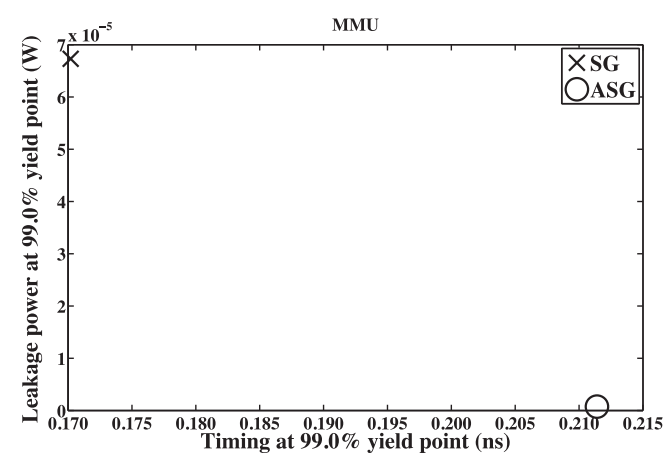

(e) Memory management unit

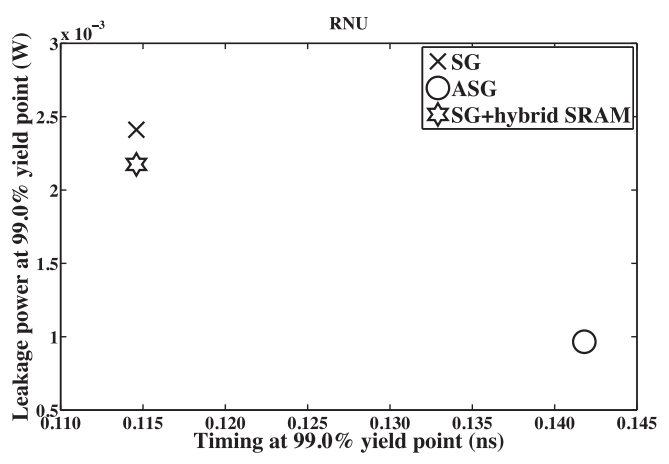

(b) Renaming unit

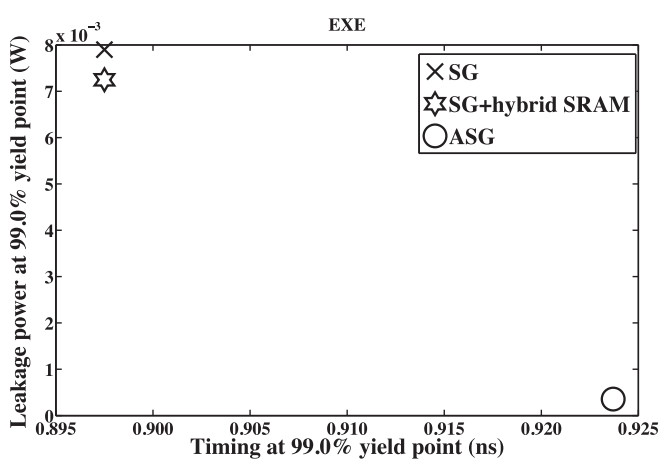

(d) Execution unit

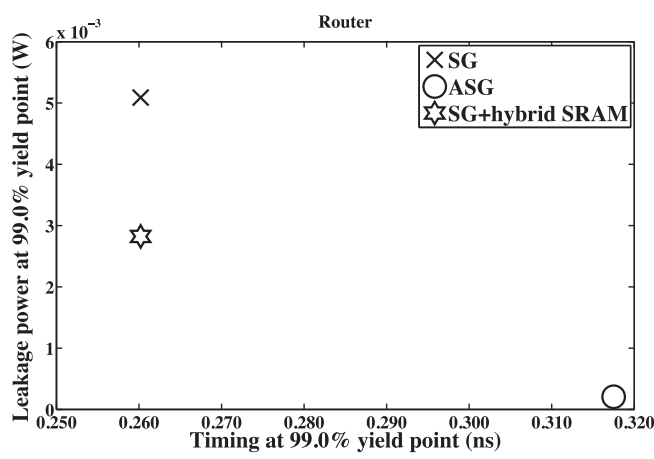

(f) Router

Fig. 9. Leakage power and timing comparisons of different core components and router configurations at $99.0 \%$ yield points. SG, ASG, and SG logic with hybrid peripheral circuit SRAM (if no SRAM is in the component, then it reduces to the SG library) denote the library used to implement the module.

and delay distributions using the core macromodels, and use the distributions to estimate the yields for each combination. We sort core-level solutions based on their leakage power and delay at $99.0 \%$ yield points. We eliminate dominated core-level solutions (having both higher leakage and delay) to reduce the search space for processor optimization. Then, we enumerate the remaining nondominated core-level candidates in tandem with candidate solutions for other components of the processor, e.g., L2 cache and memory controller. This forms the processor solution pool. We adjust 
the leakage power, dynamic power, and delay, taking into account spatial correlations between core modules and from core to core. We describe the spatial correlation model in the next section.

After spatial-correlation-based adjustments, we obtain all the processor-level solutions. We then sort these solutions according to their leakage power and delay at $99.0 \%$ yield points, and eliminate dominated solutions. Now, only non-dominated solutions remain (no processor solution has both lower leakage power and lower delay than another). Our two-step optimization method significantly improves the chances that the true globally optimal solutions are in our final solution pool. However, even after performing the above steps, the processor solution pool tends to remain large. Hence, we prune the solution space further by recognizing that some solutions have a very close configuration and performance relative to others. Therefore, we need metrics to eliminate such solutions. For any two solutions, $A=\left\{a_{1}, a_{2}, \ldots, a_{n}\right\}$ and $B=\left\{b_{1}, b_{2}, \ldots, b_{n}\right\}$, where $A$ and $B$ are processor configurations and $a_{1}-a_{n}$ and $b_{1}-b_{n}$ are corresponding power/delay characteristics, we remove $B$ from the solution pool if it satisfies the following two conditions:

(1) Tolerance condition:

$$
\forall i \in\{1,2, \ldots, n\}, \quad a_{i}<\left(1+\sigma_{1}\right) b_{i}
$$

(2) Surpass condition:

$$
\exists i \in\{1,2, \ldots, n\}, \quad a_{i}<\left(1-\sigma_{2}\right) b_{i},
$$

where $i$ indexes the value we are comparing. In our case, $n=3$, and $a_{i}$ and $b_{i}$ correspond to leakage power, dynamic power, or delay at the $99.0 \%$ yield point. We take dynamic power into account in processor solutions because dynamic power reduction becomes significant, though it is not substantial in individual modules. The tolerance condition implies that all values for $A$ (leakage power, dynamic power, and delay) are smaller than or close to (with less than $\sigma_{1}$ overhead) that of $B$. The surpass condition implies that $A$ is superior to $B$ in at least one value (at least $\sigma_{2}$ lower). The values of $\sigma_{1}$ and $\sigma_{2}$ can be tuned to control the number of final solutions. We use $\sigma_{1}=3 \%$ and $\sigma_{2}=10 \%$ in our simulations to maintain a small number (less than ten) of final optimization solutions.

\subsection{Spatial Correlation}

The McPAT-PVT processor macromodels we use do not compute the joint dynamic/leakage power or joint delay distributions of the constituent modules, since the floorplan information is not available to the model. Thus, to account for module- and core-level spatial correlations, we first need to generate the floorplan of the processor. As shown in Figure 2 under the spatial correlation model block, the connections between modules and cores are generated based on the processor configuration file. This information, together with area/power information of all components in each processor solution, is provided to HotFloorplan (Skadron et al. 2003), which is a floorplan generator that is used to produce a floorplan for every enumerated module/core combination. We then adjust the power/delay of each solution using our spatial correlation model, based on the floorplan and results from the McPAT-PVT processor model.

To model the spatial correlation between modules/cores using the leakage/dynamic power and delay distributions, we need to calculate the leakage/dynamic power covariance and delay covariance between any two components in the processor. Leakage and dynamic power can be modeled with a joint lognormal distribution and a joint normal distribution, respectively (Chang and Sapatnekar 2005a). We adopt the method of calculating the covariance of two components from Chen and Jha (2015). For ease of exposition, we restate it here: for any two modules/cores $C$ and $C^{\prime}$ with standard deviation of power consumption (leakage/dynamic) $\sigma_{P_{C}}$ and $\sigma_{P_{C^{\prime}}}$, the power covariance, $\operatorname{cov}\left(P_{C}, P_{C^{\prime}}\right)$, can be calculated from the correlation coefficient $\rho\left(P_{C}, P_{C^{\prime}}\right)$ :

$$
\operatorname{cov}\left(P_{C}, P_{C^{\prime}}\right)=\rho\left(P_{C}, P_{C^{\prime}}\right) \cdot \sigma_{P_{C}} \cdot \sigma_{P_{C^{\prime}}}
$$




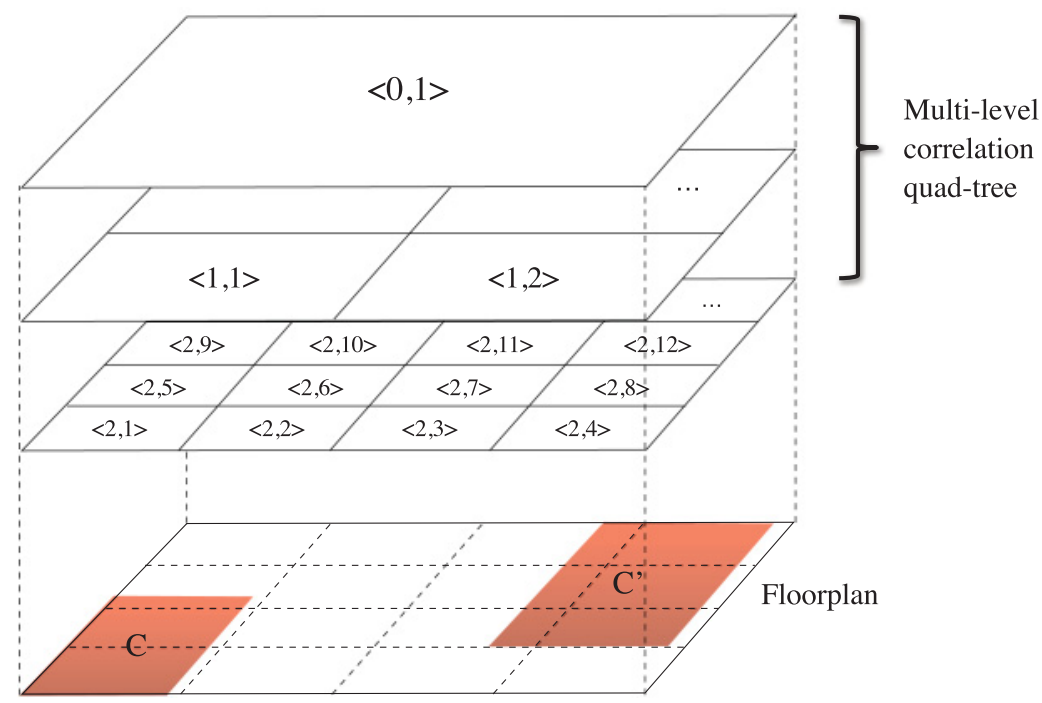

Fig. 10. Multi-level quad-tree model for spatial correlation.

where $P_{C}$ and $P_{C^{\prime}}$ represent leakage power or dynamic power of components $C$ and $C^{\prime}$, respectively. $\rho\left(P_{C}, P_{C^{\prime}}\right)$ is estimated by $\hat{\rho}\left(P_{C}, P_{C^{\prime}}\right)$ (see Equation (8)) using the multi-level quad-tree model under a sea-of-NAND2 assumption (a 2-input NAND gate is the most commonly used gate) [Agarwal et al. 2003], as shown in Figure 10. A die is hierarchically partitioned into several grid levels, where the variation of a parameter in each grid is modeled as a zero-mean random variable. Random variables in different grids or levels are assumed to be independent of each other. Hence, the topmost level models die-to-die variations, whereas lower levels model within-die variations.

$$
\hat{\rho}\left(P_{C}, P_{C^{\prime}}\right)=\operatorname{cov}\left(P_{C}, P_{C^{\prime}}\right) /\left(\hat{\sigma}_{P_{C}} \cdot \hat{\sigma}_{P_{C^{\prime}}}\right),
$$

where $\operatorname{cov}\left(P_{C}, P_{C^{\prime}}\right)$ is calculated using Equation (9); $\hat{\sigma}_{P_{C}}$ and $\hat{\sigma}_{P_{C^{\prime}}}$ are calculated using Equation (10) (Chen and Jha 2015).

$$
\begin{aligned}
\operatorname{cov}\left(P_{C}, P_{C^{\prime}}\right) & =E\left(P_{C} \cdot P_{C^{\prime}}\right)-E\left(P_{C}\right) \cdot E\left(P_{C^{\prime}}\right) \\
& =\sum_{i \in n} \sum_{g \in S_{i C}, g^{\prime} \in S_{i C^{\prime}}} \operatorname{cov}\left(P_{C, g}, P_{C^{\prime}, g^{\prime}}\right) \\
\hat{\sigma}_{P_{C\left(C^{\prime}\right)}}= & \sqrt{E\left(P_{C\left(C^{\prime}\right)}^{2}\right)-E\left(P_{C\left(C^{\prime}\right)}\right)^{2}} \\
= & \sqrt{\sum_{i \in n} \sum_{g, g^{\prime} \in S_{i C\left(C^{\prime}\right)}} \operatorname{cov}\left(P_{C\left(C^{\prime}\right), g}, P_{C\left(C^{\prime}\right), g^{\prime}}\right)},
\end{aligned}
$$

where $n$ denotes the number of levels used in the multi-level correlation quad-tree and $S_{i C}$ denotes the set of grids in level $i$ covered by $C$. For example, in Figure 10, $S_{2 C}$ covers $\{\langle 2,1\rangle,\langle 2,5\rangle,\langle 2,9\rangle\}$ and $S_{2 C^{\prime}}$ covers $\{\langle 2,7\rangle,\langle 2,8\rangle,\langle 2,11\rangle,\langle 2,12\rangle,\langle 2,15\rangle,\langle 2,16\rangle\} . P_{C, g}$ denotes the power consumption of grid $g$ in component $C$, which is computed based on the sea-of-NAND2 assumption:

$$
P_{C, g}=\alpha_{C, g} \cdot N \cdot P_{N A N D 2, g},
$$

where $\alpha_{C, g}$ denotes the portion of grid $g$ covered by component $C, N$ is the number of NAND2 gates in each grid, and $P_{N A N D 2, g}$ is the power consumption of a NAND2 gate (Chen and Jha 2015). 
Table 3. Processor Configuration Parameters

\begin{tabular}{cc}
\hline Parameter & Value (single-core/8-core) \\
\hline Processor design & Alpha/Niagara \\
Number of cores & $1 / 8$ \\
Fetch/Decode/Issue/Commit width per core & $4 / 1$ \\
Number of ALUs per core & $4 / 1$ \\
Number of MULs per core & $0 / 1$ \\
Number of FPUs per core & $1 / 0.125$ \\
Load/store buffer size & 32 \\
L1 instruction cache capacity $(K B)$ & $64 / 16$ \\
L1 data cache capacity $(K B)$ & $64 / 8$ \\
L2 cache capacity $(M B)$ & $1.75 / 3$ \\
L1 instruction cache block size $(B)$ & $16 / 32$ \\
L1 data cache block size $(B)$ & $16 / 16$ \\
L2 cache block size $(B)$ & $16 / 64$ \\
L1 cache associativity & $2 / 4$ \\
L2 cache associativity & $8 / 16$ \\
Cache access model & $\mathrm{UCA}$ \\
Technology & $22 \mathrm{~nm}$ FinFET \\
Frequency $(G H z)$ & 1.2 \\
Temperature $(K)$ & $358 / 368$ \\
$V_{D D}(V)$ & 0.9 \\
$3 \sigma / \mu$ & $10 \%$ \\
\hline
\end{tabular}

Plugging Equation (11) into Equations (9) and (10) transfers côv $\left(P_{C}, P_{C^{\prime}}\right)$ and $\hat{\sigma}_{P_{C\left(C^{\prime}\right)}}$ as functions of $\operatorname{cov}\left(P_{N A N D 2, g}, P_{N A N D 2, g^{\prime}}\right)$, which is calculated as follows:

$$
\operatorname{cov}\left(P_{N A N D 2, g}, P_{N A N D 2, g^{\prime}}\right)=\sum_{p i \in \vec{p}}\left[\frac{\partial P}{\partial p i}\right]_{n o m} \cdot r\left(g, g^{\prime}\right) \cdot \operatorname{var}(p i),
$$

where $\vec{p}$ is the set of process parameters, e.g., $L_{G}, T_{X I}$, and so on, and $r$ is a covariance factor depending on locations of grids $g$ and $g^{\prime}$.

The joint distribution of delay is calculated using Equations (7) and (8) with power replaced by delay.

\section{SIMULATION RESULTS}

In this section, we report statistical optimization results for a single-core processor configured based on Alpha 21364 (Jain et al. 2001) and an 8-core CMP configured based on Niagara (Kongetira et al. 2005) using our proposed dual device-type assignment method. The configurations of the two processors are shown in Table 3. There is only one FPU in the 8-core processor, hence, the average number of FPUs per core is 0.125 . Since temperature can settle at any value, we model it as an independent parameter with no correlation to process-voltage variations. We assume the operating temperature of the single-core processor to be $358 \mathrm{~K}$, and $10 \mathrm{~K}$ higher for the 8-core processor, since it will likely have a higher power density. Both processors are implemented with $22 \mathrm{~nm}$ FinFET technology and operate at a clock rate of $1.2 \mathrm{GHz}$. We assume a normal distribution for all process parameters and $V_{D D}$, and the $3 \sigma$ values of their distributions are set to $10 \%$ of their nominal values, which is a typical assumption made in statistical analysis of such circuits. 
Table 4. Single-Core Processor Optimization Results

\begin{tabular}{|c|c|c|c|c|}
\hline Solution & SG baseline & $\# 1$ & $\# 2$ & $\# 3$ \\
\hline Instruction Fetch Unit & SG & ASG & ASG & ASG \\
\hline Renaming Unit & SG & ASG & ASG & ASG \\
\hline Load-store Unit & SG & ASG & ASG & ASG \\
\hline Memory Management Unit & SG & ASG & ASG & ASG \\
\hline Execution Unit & SG & ASG & ASG & ASG \\
\hline Pipeline Register & SG & ASG & SG & SG \\
\hline L1 Cache & SG & SG & SG & SG \\
\hline L2 Cache & SG & hybrid SRAM & ASG & ASG \\
\hline Second-level Directory & SG & hybrid SRAM & hybrid SRAM & ASG \\
\hline Memory Controller & SG & ASG & ASG & ASG \\
\hline Area $\left(m^{2}\right)$ & 4.4968 & 4.5686 & 4.7744 & 4.7756 \\
\hline Total Power $(W)$ & 1.4418 & 1.1486 & 1.1544 & 1.1557 \\
\hline Leakage Power Mean $(W)$ & 0.3358 & 0.0473 & 0.0373 & 0.0372 \\
\hline Leakage Power Sigma $(W)$ & 0.0996 & 0.0080 & 0.0067 & 0.0067 \\
\hline 99.0\% Leakage Power Yield Point $(W)$ & 0.6323 & 0.0689 & 0.0556 & 0.0555 \\
\hline Dynamic Power Mean $(W)$ & 1.1060 & 1.1013 & 1.1171 & 1.1185 \\
\hline Dynamic Power Sigma $(W)$ & 0.0360 & 0.0084 & 0.0088 & 0.0088 \\
\hline 99.0\% Dynamic Power Yield Point $(W)$ & 1.1897 & 1.1210 & 1.1371 & 1.1390 \\
\hline Minimum Clock Period Mean $(n s)$ & 0.833 & 0.833 & 0.833 & 0.833 \\
\hline Minimum Clock Period Sigma $(n s)$ & 0.028 & 0.027 & 0.027 & 0.027 \\
\hline 99.0\% Timing Yield Point $(n s)$ & 0.898 & 0.896 & 0.896 & 0.896 \\
\hline
\end{tabular}

This section consists of two parts. We first discuss our optimization results for the two processors and improvements over the SG FinFET baseline implementations. Then, we show optimization results without taking into account module- and core-level spatial correlations, and demonstrate the importance of considering spatial correlations at the module and core levels.

\subsection{Statistical Optimization Results}

Tables 4 and 5 show the optimization results for the single-core and 8-core processors, respectively, along with the results for the SG baseline processors. The SG implementations are selected as the baselines because SG devices are the standard ones used in modern FinFET processors. The two processors have three and seven globally optimal solutions, respectively. The dual device-type assignment of various components of the processors is also shown in the tables.

For the single-core processor, solution number 3 has the lowest leakage power at $99.0 \%$ leakage yield point (i.e, it has the highest leakage yield): $0.0555 \mathrm{~W}$. It is $91.2 \%$ smaller compared to that of the SG baseline, in which the whole processor is implemented with only SG FinFETs. The leakage yield at the nominal value of the SG baseline leakage power (at $0.3358 \mathrm{~W}$ ) is increased by $44.2 \%$. Solution number 1 has the lowest dynamic power at $99.0 \%$ dynamic power yield point: $1.1210 \mathrm{~W}$, which is $4.3 \%$ lower relative to that of the SG baseline. The dynamic power yield is increased by $21.2 \%$ at the nominal value of the SG baseline dynamic power (at $1.1060 \mathrm{~W}$ ). All the three globally optimal solutions meet the target clock frequency $(1.2 \mathrm{GHz})$ with a smaller standard deviation, hence, they have a higher timing yield. They all dominate the baseline processor in leakage power yield, dynamic power yield, and timing yield, with an area overhead ranging from $1.6 \%$ to $6.2 \%$. This is because in our optimized solutions, SG modules are replaced by either ASG modules or hybrid SRAM modules on off-critical paths to reduce power consumption, and SG modules are 
Table 5. 8-core CMP Optimization Results

\begin{tabular}{|c|c|c|c|c|}
\hline Solution & SG baseline & $\# 1$ & $\# 2$ & \#3 \\
\hline Instruction Fetch Unit & SG & ASG SRAM + SG logic & ASG & ASG \\
\hline Renaming Unit & SG & hybrid SRAM + SG logic & hybrid SRAM + SG logic & hybrid SRAM + SG logic \\
\hline Load-store Unit & SG & hybrid SRAM + SG logic & ASG & ASG \\
\hline Memory Management Unit & SG & ASG & ASG & ASG \\
\hline Execution Unit & SG & ASG & ASG & ASG \\
\hline Pipeline Register & SG & ASG & SG & SG \\
\hline L1 Cache & SG & SG & SG & SG \\
\hline L2 Cache & SG & hybrid SRAM & ASG & ASG \\
\hline First-level Directory & SG & SG & hybrid SRAM & hybrid SRAM \\
\hline NoC & SG & ASG & ASG & hybrid SRAM + SG logic \\
\hline Flash/SSD Controllers & SG & SG & $\overline{A S G}$ & ASG \\
\hline Network Interface Unit & SG & SG & ASG & SG \\
\hline Peripheral Component Interconnect Express & SG & SG & SG & ASG \\
\hline Area $\left(\mathrm{mm}^{2}\right)$ & 7.8481 & 8.0345 & 8.3735 & 8.3735 \\
\hline Total Power $(W)$ & 4.0419 & 3.1812 & 3.4100 & 3.4108 \\
\hline Leakage Power Mean $(W)$ & 1.6453 & 0.9954 & 0.9846 & 0.9845 \\
\hline Leakage Power Sigma $(W)$ & 0.4769 & 0.2801 & 0.2474 & 0.2474 \\
\hline 99.0\% Leakage Power Yield Point $(W)$ & 3.0583 & 1.8203 & 1.6974 & 1.6973 \\
\hline Dynamic Power Mean $(W)$ & 2.3966 & 2.1858 & 2.4255 & 2.4262 \\
\hline Dynamic Power Sigma $(W)$ & 0.0717 & 0.0245 & 0.0369 & 0.0369 \\
\hline $99.0 \%$ Dynamic Power Yield Point $(W)$ & 2.5634 & 2.2428 & 2.5114 & 2.5121 \\
\hline Minimum Clock Period Mean $(n s)$ & 0.833 & 0.833 & 0.833 & 0.833 \\
\hline Minimum Clock Period Sigma $(n s)$ & 0.132 & 0.099 & 0.099 & 0.101 \\
\hline $99.0 \%$ Timing Yield Point $(n s)$ & 1.141 & 1.064 & 1.064 & 1.069 \\
\hline
\end{tabular}

\begin{tabular}{|c|c|c|c|c|}
\hline Solution & $\# 4$ & $\# 5$ & $\# 6$ & $\# 7$ \\
\hline Instruction Fetch Unit & ASG & ASG SRAM + SG logic & ASG SRAM + SG logic & ASG \\
\hline Renaming Unit & hybrid SRAM + SG logic & hybrid SRAM + SG logic & hybrid SRAM + SG logic & ASG \\
\hline Load-store Unit & ASG & hybrid SRAM + SG logic & hybrid SRAM + SG logic & ASG \\
\hline Memory Management Unit & ASG & \begin{tabular}{|c|} 
ASG \\
\end{tabular} & SG & ASG \\
\hline Execution Unit & ASG & ASG & ASG & ASG \\
\hline Pipeline Register & ASG & ASG & ASG & SG \\
\hline L1 Cache & SG & SG & SG & SG \\
\hline L2 Cache & ASG & hybrid SRAM & ASG & ASG \\
\hline First-level Directory & hybrid SRAM & ASG & hybrid SRAM & hybrid SRAM \\
\hline NoC & ASG & ASG & ASG & \begin{tabular}{|c|} 
ASG \\
\end{tabular} \\
\hline Flash/SSD Controllers & ASG & ASG & ASG & ASG \\
\hline \begin{tabular}{|c|} 
Network Interface Unit \\
\end{tabular} & ASG & SG & ASG & ASG \\
\hline Peripheral Component Interconnect Express & ASG & SG & SG & ASG \\
\hline Area $\left(\mathrm{mm}^{2}\right)$ & 8.3733 & 8.1380 & 8.3428 & 8.4230 \\
\hline Total Power $(W)$ & 3.4119 & 3.1770 & 3.4164 & 3.4790 \\
\hline Leakage Power Mean $(W)$ & 0.9831 & 0.9904 & 0.9850 & 0.9818 \\
\hline Leakage Power Sigma $(W)$ & 0.2472 & 0.2795 & 0.2483 & 0.2469 \\
\hline 99.0\% Leakage Power Yield Point $(W)$ & 1.6954 & 1.8140 & 1.7007 & 1.6932 \\
\hline Dynamic Power Mean $(W)$ & 2.4289 & 2.1866 & 2.4314 & 2.4971 \\
\hline Dynamic Power Sigma $(W)$ & 0.0369 & 0.0245 & 0.0369 & 0.0369 \\
\hline $99.0 \%$ Dynamic Power Yield Point $(W)$ & 2.5148 & 2.2436 & 2.5175 & 2.5830 \\
\hline Minimum Clock Period Mean $(n s)$ & 0.833 & 0.833 & 0.833 & 0.833 \\
\hline Minimum Clock Period Sigma $(n s)$ & 0.102 & 0.088 & 0.088 & 0.090 \\
\hline 99.0\% Timing Yield Point $(n s)$ & 1.069 & 1.038 & 1.038 & 1.042 \\
\hline
\end{tabular}

only used on critical paths to maintain a high clock rate, meeting the timing yield constraint. Detailed module configurations are also shown in the table. SG (ASG) entries imply that the whole module is implemented with just SG (ASG) FinFETs, whereas "hybrid SRAM" implies that the SRAM in the module uses the hybrid model mentioned earlier.

The L1 cache is a critical component of the processors that determines the clock rate. Thus, all three optimized solutions use SG FinFETs for the L1 cache implementation to meet the $1.2 \mathrm{GHz}$ target clock rate. Other functional units (instruction fetch unit, renaming unit, load-store unit, memory management unit, execution unit, etc.) are implemented using ASG FinFETs to reduce leakage power. Various configurations are used for the pipeline register, L2 cache, and secondlevel directory to reduce leakage power while meeting the clock period constraint. The standard 
Table 6. Single-core Processor Optimization Results When Module- and Core-Level Spatial Correlations Are Not Taken into Account

\begin{tabular}{|c|c|c|c|c|c|}
\hline Solution Number & $\# 1$ & $\# 2$ & \#3 & $\# 4$ & \#5 \\
\hline Instruction Fetch Unit & ASG & hybrid SRAM + SG logic & ASG & SG & ASG \\
\hline Renaming Unit & ASG & hybrid SRAM + SG logic & ASG & hybrid SRAM + SG logic & ASG \\
\hline Load-store Unit & ASG & hybrid SRAM + SG logic & ASG & hybrid SRAM + SG logic & ASG \\
\hline Memory Management Unit & ASG & SG & ASG & ASG & $\mathrm{ASG}$ \\
\hline Execution Unit & ASG & hybrid SRAM + SG logic & ASG & ASG & ASG \\
\hline Pipeline Register & ASG & SG & ASG & ASG & ASG \\
\hline L1 Cache & ASG & hybrid SRAM & SG & SG & SG \\
\hline L2 Cache & ASG & hybrid SRAM & ASG & SG & ASG \\
\hline Second-level Directory & ASG & hybrid SRAM & hybrid SRAM & hybrid SRAM & ASG \\
\hline Memory Controller & ASG & hybrid SRAM + SG logic & ASG & ASG & ASG \\
\hline Area $\left(\mathrm{mm}^{2}\right)$ & 4.7976 & 4.4969 & 4.5686 & 4.7744 & 4.7756 \\
\hline Total Power $(W)$ & 1.1673 & 1.1176 & 1.1657 & 1.1447 & 1.1457 \\
\hline Leakage Power Mean $(W)$ & 0.0256 & 0.0918 & 0.0660 & 0.0276 & 0.0272 \\
\hline Leakage Power Sigma $(W)$ & 0.0058 & 0.0250 & 0.0179 & 0.0064 & 0.0062 \\
\hline 99.0\% Leakage Power Yield Point $(W)$ & 0.0420 & 0.1649 & 0.1183 & 0.0458 & 0.0447 \\
\hline Dynamic Power Mean $(W)$ & 1.1417 & 1.0258 & 1.0997 & 1.1171 & 1.1185 \\
\hline Dynamic Power Sigma $(W)$ & 0.0408 & 0.0339 & 0.0374 & 0.0384 & 0.0385 \\
\hline 99.0\% Dynamic Power Yield Point $(W)$ & 1.2366 & 1.1047 & 1.1865 & 1.2065 & 1.2080 \\
\hline Minimum Clock Period Mean ( $n s)$ & 0.833 & 0.833 & 0.833 & 0.833 & 0.833 \\
\hline Minimum Clock Period Sigma $(n s)$ & 0.027 & 0.027 & 0.027 & 0.027 & 0.027 \\
\hline $99.0 \%$ Timing Yield Point $(n s)$ & 0.896 & 0.896 & 0.896 & 0.896 & 0.896 \\
\hline
\end{tabular}

deviations of the minimum clock period of optimized solutions are also slightly smaller than that of the SG baseline because of smaller $R C$ delay variation.

For the 8-core processor, solution number 7 has the lowest $99.0 \%$ leakage power yield point, $1.6932 \mathrm{~W}$, which is $44.6 \%$ lower relative to that of the SG baseline processor. The leakage power yield at the nominal value of the baseline leakage power is increased by $43.0 \%$. This is because most of its components are implemented using ASG FinFETs (all functional units inside the core, NoC, and peripheral controllers). Solution number 1 has the lowest $99.0 \%$ dynamic power yield point, which is $12.5 \%$ lower relative to that of the baseline, and the dynamic power yield at the nominal value of the baseline dynamic power is increased by $50.0 \%$. All solutions meet the target clock rate with smaller standard deviations and the area overheads range from $2.4 \%$ to $7.3 \%$. Module configurations are the same for each core in the 8-core processor, since it is a homogeneous-core CMP. As in the case of the single-core processor and for the same reason, the L1 cache is implemented with SG FinFETs. Dynamic power does not improve as much as leakage power, since we mainly focus on leakage and timing yield enhancements in local optimization.

\subsection{Discussion of Module- and Core-Level Spatial Correlations}

Table 6 shows the optimized results for the single-core processor when module- and core-level spatial correlations are not taken into account. These solutions have different component configurations than when module- and core-level spatial correlations are considered. Compared with the results shown in Table 4, these optimized results have lower $99.0 \%$ leakage and dynamic power yield points: solution number 1 has the lowest leakage yield point, $0.0420 \mathrm{~W}$, where the whole processor is implemented with ASG FinFETs. The lowest dynamic power yield point is obtained in solution number 2, at $1.1047 \mathrm{~W}$, in which all SRAMs are hybrid and the rest of the processor is implemented with SG FinFETs.

Although these results have lower leakage/dynamic power yield points (i.e., higher leakage/ dynamic power yields) at the same timing yield, they are unrealistic for two reasons. First, the computation of leakage/dynamic power yields excludes module/core-level correlations. Since PVT 
variations impact different modules/cores similarly when they are close to each other on the IC, and less so when they are far away from each other, excluding these spatial correlations overestimates the leakage/dynamic power yields. Second, timing yield is also overestimated in the same manner, which, in turn, leads to further overestimation of leakage/dynamic yields. This is because of timing yield slack (timing yield achieved minus required yield) due to the overestimation enables more lower-power, lower-performance components to be employed in order to reduce leakage/dynamic power (thus increasing power yields), while still satisfying the timing yield constraint. This is why a processor implemented with only ASG FinFETs is one of the optimized solutions when system-level spatial correlations are not considered, but not when spatial correlations are considered: it either does not satisfy the delay constraint with its low-power configuration or the power consumption goes up when the clock period is shrunk (through upsizing of logic gates). Thus, including spatial correlations at all levels (from the standard cell to module and core) is important and necessary for accurate yield estimation.

\section{DISCUSSION}

The simulation results presented in Section 4 show the efficacy of our proposed architectural statistical optimization framework using dual device-type assignment: ASG FinFETs are used to improve the power yields while SG FinFETs are used to maintain the timing yield. We perform statistical optimization in a bottom-up fashion: starting from the lowest functional unit level in the processor hierarchy in Figure 4, simulating the functional units with both SG and ASG (hybrid) configurations and selecting the locally optimal candidates for upper-level optimizations. Modules at the higher level are simulated and optimized using lower-level optimal candidates with the SG, ASG, and hybrid libraries. This bottom-up treatment ensures the optimality of the final solutions, which only consist of locally optimal candidates. The tradeoffs among different locally optimized modules are also taken into consideration as we perform an exhaustive enumeration of all combinations of locally optimal candidates at core and processor levels, which guarantees that the yield improvement in one domain or of one module does not harm the yield in another domain or of another module.

\section{CONCLUSION}

In this article, we presented a dual device-type assignment-based statistical optimization method for FinFET-based CMPs under PVT variations that takes spatial correlations into account at all levels of the design hierarchy. The method uses McPAT-PVT and FinCANON, which contain a FinFET design library for modeling standard cells and SRAM cells, and macromodels for cache, NoC, and other modules in the architecture hierarchy. We described a two-step optimization method that first optimizes each architectural component locally with dual device-type assignment to find non-dominated configuration candidates, then enumerates all combinations of these candidates and selects globally optimized solutions for the processor. We included a module/core-level spatial correlation model to improve yield estimation accuracy. We performed statistical optimizations for a single-core processor and an 8-core CMP. Results show that our optimization method can improve leakage and dynamic power yields with no timing yield loss and only a small area overhead. It considers tradeoffs across different modules inside the processor. We also justified the importance of taking into account module/core-level spatial correlations for accurate yield estimation.

\section{REFERENCES}

A. Agarwal, D. Blaauw, and V. Zolotov. 2003. Statistical timing analysis for intra-die process variations with spatial correlations. In Proc. Int. Conf. Comput.-Aided Design. 900-907.

A. G. Akkala, R. Venkatesan, A. Raghunathan, and K. Roy. 2016. Asymmetric underlapped sub-10-nm n-FinFETs for highspeed and low-leakage 6T SRAMs. IEEE Trans. Electron Devices 63, 3 (Mar. 2016), 1034-1040. 
M. G. Bardon, P. Raghavan, G. Eneman, P. Schuddinck, M. Dehan, A. Mercha, A. Thean, D. Verkest, and A. Steegen. 2014. Group IV channels for 7nm FinFETs: Performance for SoCs power and speed metrics. In Proc. Symp. VLSI Technology. $1-2$.

D. K. Beece, C. Visweswariah, J. Xiong, and V. Zolotov. 2014. Transistor sizing of custom high-performance digital circuits with parametric yield considerations. Optimization and Eng. 15, 1 (July 2014), 217-241.

A. N. Bhoj and N. K. Jha. 2013. Design of logic gates and flip-flops in high-performance FinFET technology. IEEE Trans. VLSI Syst. 21, 11 (Nov. 2013), 1975-1988.

A. N. Bhoj and N. K. Jha. 2014. Parasitics-aware design of symmetric and asymmetric gate-workfunction FinFET SRAMs. IEEE Trans. VLSI Syst. 22, 3 (Mar. 2014), 548-561.

A. N. Bhoj, R. V. Joshi, and N. K. Jha. 2013. 3-D-TCAD-based parasitic capacitance extraction for emerging multigate devices and circuits. IEEE Trans. VLSI Syst. 21, 11 (Nov. 2013), 2094-2105.

C. Bienia, S. Kumar, J. P. Singh, and K. Li. 2008. The PARSEC benchmark suite: Characterization and architectural implications. In Proc. ACM Int. Conf. Parallel Archit. Compilation Techniques. 72-81.

N. Binkert, B. Beckmann, G. Black, S. K. Reinhardt, A. Saidi, A. Basu, J. Hestness, D. R. Hower, T. Krishna, and S. Sardashti. 2011. The gem5 simulator. ACM SIGARCH Comput. Archit. News 39, 2 (May 2011), 1-7.

A. Carlson, Z. Guo, S. Balasubramanian, L. T. Pang, T. J. King Liu, and B. Nikolic. 2006. FinFET SRAM with enhanced read/write margins. In Proc. IEEE Int. SOI Conf. 105-106.

H. Chang and S. S. Sapatnekar. 2005a. Full-chip analysis of leakage power under process variations, including spatial correlations. In Proc. ACM Design Autom. Conf. 523-528.

H. Chang and S. S. Sapatnekar. 2005b. Statistical timing analysis under spatial correlations. IEEE Trans. Comput.-Aided Design Integr. Circuits Syst. 24, 9 (Sept. 2005), 1467-1482.

S. Chaudhuri and N. K. Jha. 2011. 3D vs. 2D analysis of FinFET logic gates under process variations. In Proc. IEEE Int. Conf. Computer Design. 435-436.

S. M. Chaudhuri. and N. K. Jha. 2014. 3D vs. 2D device simulation of FinFET logic gates under PVT variations. ACM 7 . Emerg. Technol. Comput. Syst. 10, 3 (May 2014), 26:1-26:19.

X. Chen and N. K. Jha. 2014. Ultra-low-leakage chip multiprocessor design with hybrid FinFET logic styles. ACM f. Emerg. Technol. Comput. Syst. 11, 1 (Oct. 2014), 3:1-3:16.

X. Chen and N. K. Jha. 2015. gem5-PVT: A framework for FinFET system simulation under PVT variations. ACM f. Emerg. Technol. Comput. Syst. 12, 3 (Sept. 2015), 28:1-28:19.

K. Cheng, P. Hashemi, A. Khakifirooz, and A. Reznicek. 2016. Dual work function integration for stacked FinFET. Retrieved from http://www.freepatentsonline.com/y2016/0336235.html.

J. H. Choi, J. Murthy, and K. Roy. 2007. The effect of process variation on device temperature in FinFET circuits. In Proc. IEEE/ACM Int. Conf. Comput.-Aided Design. 747-751.

C. H. Chou, C. C. Hsu, W. K. Yeh, S. S. Chung, and C. H. Chien. 2016. 3D-TCAD simulation study of the contact all around T-FinFET structure for $10 \mathrm{~nm}$ metal-oxide-semiconductor field-effect transistor. In Proc. IEEE Silicon Nanoelectronics Wkshp. 190-191.

C. E. Clark. 1961. The greatest of a finite set of random variables. Operations Research 9, 2 (1961), 145-162.

N. A. Conos, S. Meguerdichian, S. Wei, and M. Potkonjak. 2013. Maximizing yield in near-threshold computing under the presence of process variation. In Proc. Int. Wkshp. Power and Timing Modeling, Optimization and Simulation. 1-8.

X. Dai and N. K. Jha. 2017. Improving convergence and simulation time of quantum hydrodynamic simulation: Application to extraction of best 10-nm FinFET parameter values. IEEE Trans. VLSI Syst. 25, 1 (Jan. 2017), 319-329.

A. Dutta, K. Koley, S. K. Saha, and C. K. Sarkar. 2016. Physical insights into electric field modulation in dual-k spacer asymmetric underlap FinFET. IEEE Trans. Electron Devices 63, 8 (Aug. 2016), 3019-3027.

S. Garg and D. Marculescu. 2009a. 3D-GCP: An analytical model for the impact of process variations on the critical path delay distribution of 3D ICs. In Proc. IEEE Int. Symp. Quality Electron. Design. 147-155.

S. Garg and D. Marculescu. 2009b. System-level process variability analysis and mitigation for 3D MPSoCs. In Proc. IEEE Design, Autom. \& Test Europe Conf. \& Exhibition. 604-609.

M. Guillorn, J. Chang, A. Bryant, N. Fuller, O. Dokumaci, X. Wang, J. Newbury, K. Babich, J. Ott, B. Haran, R. Yu, C. Lavoie, D. Klaus, Y. Zhang, E. Sikorski, W. Graham, B. To, M. Lofaro, J. Tornello, D. Koli, B. Yang, A. Pyzyna, D. Neumeyer, M. Khater, A. Yagishita, H. Kawasaki, and W. Haensch. 2008. FinFET performance advantage at 22nm: An AC perspective. In Proc. Symp. VLSI Technol. 12-13.

Z. Guo, S. Balasubramanian, R. Zlatanovici, T. J. King, and B. Nikolić. 2005. FinFET-based SRAM design. In Proc. ACM Int. Symp. Low Power Electron. Design. 2-7.

M. A. Horowitz. 1983. Timing Models for MOS Circuits. Ph.D. Dissertation. Technical Report, Stanford University.

M. M. Hussain, C. Smith, P. Kalra, J. Yang, G. Gebara, B. Sassman, P. Kirsch, P. Majhi, S. Song, R. Harris, H. Tseng, and R. Jammy. 2007. Dual work function high-k/metal gate CMOS FinFETs. In Proc. European Solid State Device Research Conf. 207-209. 
E. J. Hwang, W. Kim, and Y. H. Kim. 2013. Timing yield slack for timing yield-constrained optimization and its application to statistical leakage minimization. IEEE Trans. VLSI Syst. 21, 10 (Oct. 2013), 1783-1796.

ITRS. 2013. International Technology Roadmap for Semiconductors. Retrieved from http://www.itrs.net.

A. Jain, W. Anderson, T. Benninghoff, D. Berucci, M. Braganza, J. Burnetie, T. Chang, J. Eble, R. Faber, O. Gowda, and others. 2001. A $1.2 \mathrm{GHz}$ Alpha microprocessor with $44.8 \mathrm{~GB} / \mathrm{s}$ chip pin bandwidth. In Proc. IEEE Int. Solid-State Circuits Conf. $240-241$.

R. V. Joshi, K. Kim, R. Q. Williams, E. J. Nowak, and C. T. Chuang. 2007. A high-performance, low leakage, and stable SRAM row-based back-gate biasing scheme in FinFET technology. In Proc. IEEE Int. Conf. VLSI Design. 665-672.

J. Kedzierski, D. M. Fried, E. J. Nowak, T. Kanarsky, J. H. Rankin, H. Hanafi, W. Natzle, D. Boyd, Y. Zhang, R. A. Roy, J. Newbury, C. Yu, Q. Yang, P. Saunders, C. P. Willets, A. Johnson, S. P. Cole, H. E. Young, N. Carpenter, D. Rakowski, B. A. Rainey, P. E. Cottrell, M. Ieong, and H. S. P. Wong. 2001. High-performance symmetric-gate and CMOS-compatible $V_{t}$ asymmetric-gate FinFET devices. In Proc. Int. Electron Devices Meeting. 19.5.1-19.5.4.

J. H. Kim, W. Kim, and Y. H. Kim. 2015. Efficient statistical timing analysis using deterministic cell delay models. IEEE Trans. VLSI Syst. 23, 11 (Nov. 2015), 2709-2713.

Y. B. Kim, Y. B. Kim, and F. Lombardi. 2008. Low power 8T SRAM using 32nm independent gate FinFET technology. In Proc. IEEE Int. SOC Conf. 247-250.

P. Kongetira, K. Aingaran, and K. Olukotun. 2005. Niagara: A 32-way multithreaded SPARC processor. IEEE Micro 25, 2 (June 2005), 21-29.

C. Y. Lee and N. K. Jha. 2014. FinCANON: A PVT-aware integrated delay and power modeling framework for FinFET-based caches and on-chip networks. IEEE Trans. VLSI Syst. 22, 5 (May 2014), 1150-1163.

L. Mathew, M. Sadd, B. E. White, A. Vandooren, S. Dakshina-Murthy, J. Cobb, T. Stephens, R. Mora, D. Pham, J. Conner, T. White, Z. Shi, A. V. Y. Thean, A. Barr, M. Zavala, J. Schaeffer, M. J. Rendon, D. Sing, M. Orlowski, B. Y. Nguyen, and J. Mogab. 2003. FinFET with isolated $\mathrm{n}+$ and $\mathrm{p}+$ gate regions strapped with metal and polysilicon. In Proc. IEEE Int. Conf. SOI. 109-110.

P. Mishra, A. N. Bhoj, and N. K. Jha. 2010. Die-level leakage power analysis of FinFET circuits considering process variations. In Proc. Int. Symp. Quality Electron. Design. 347-355.

M. Mosaffa, S. Mohammadi, and S. Safari. 2016. Statistical analysis of asynchronous pipelines in presence of process variation using formal models. Integration, the VLSI fournal 55 (Sept. 2016), 98-117.

D. Nguyen, A. Davare, M. Orshansky, D. Chinnery, B. Thompson, and K. Keutzer. 2003. Minimization of dynamic and static power through joint assignment of threshold voltages and sizing optimization. In Proc. Int. Symp. Low Power Electron. Design. 158-163.

M. Raji, A. Tajary, B. Ghavami, H. Pedram, and H. R. Zarandi. 2011. Statistical leakage power optimization of asynchronous circuits considering process variations. In Integrated Circuit and System Design: Power and Timing Modeling, Optimization, and Simulation. Springer, 126-136.

K. Roy, H. Mahmoodi, S. Mukhopadhyay, H. Ananthan, A. Bansal, and T. Cakici. 2006. Double-gate SOI devices for lowpower and high-performance applications. In Proc. IEEE/ACM Int. Conf. Comput.-Aided Design. 217-224.

S. R. Sarangi, B. Greskamp, R. Teodorescu, J. Nakano, A. Tiwari, and J. Torrellas. 2008. VARIUS: A model of process variation and resulting timing errors for microarchitects. IEEE Trans. Semicond. Manuf. 21, 1 (Feb. 2008), 3-13.

A. K. Singh, K. He, C. Caramanis, and M. Orshansky. 2014. Modeling and optimization techniques for yield-aware SRAM post-silicon tuning. IEEE Trans. Comput.-Aided Design Integr. Circuits Syst. 33, 8 (Aug. 2014), 1159-1167.

S. Sinha, L. Shifren, V. Chandra, B. Cline, G. Yeric, R. Aitken, B. Cheng, A. R. Brown, C. Riddet, C. Alexandar, C. Millar, and A. Asenov. 2015. Circuit design perspectives for Ge FinFET at 10nm and beyond. In Proc. Int. Symp. Quality Electron. Design. 57-60.

K. Skadron, M. R. Stan, W. Huang, S. Velusamy, K. Sankaranarayanan, and D. Tarjan. 2003. Temperature-aware microarchitecture. ACM SIGARCH Comput. Archit. News 31, 2 (June 2003), 2-13.

A. Srivastava, D. Sylvester, and D. Blaauw. 2004. Power minimization using simultaneous gate sizing, dual-Vdd and dual-Vth assignment. In Proc. ACM Design Autom. Conf. 783-787.

Synopsys. 2016a. Design Compiler. Retrieved from http://www.synopsys.com/Tools/Implementation/RTLSynthesis/ DesignCompiler/Pages/default.aspx.

Synopsys. 2016b. Synopsys, Process and Device Simulation Tools to Accelerate Innovation. Retrieved from http://www. synopsys.com/tools/tcad.

A. Tang and N. K. Jha. 2016. GenFin: Genetic algorithm-based multiobjective statistical logic circuit optimization using incremental statistical analysis. IEEE Trans. VLSI Syst. 24, 3 (Mar. 2016), 1126-1139.

A. Tang, Y. Yang, C. Y. Lee, and N. K. Jha. 2014. McPAT-PVT: Delay and power modeling framework for FinFET processor architectures under PVT variations. IEEE Trans. VLSI Syst. 23, 9 (Sept. 2014), 1616-1627.

H. S. Wang, X. Zhu, L. S. Peh, and S. Malik. 2002. Orion: A power-performance simulator for interconnection networks. In Proc. IEEE/ACM Int. Symp. Microarchitecture. 294-305. 
S. J. E. Wilton and N. P. Jouppi. 1996. CACTI: An enhanced cache access and cycle time model. IEEE f. Solid-State Circuits 31, 5 (Aug. 1996), 677-688.

C. C. Wu, D. W. Lin, A. Keshavarzi, C. H. Huang, C. T. Chan, C. H. Tseng, C. L. Chen, C. Y. Hsieh, K. Y. Wong, M. L. Cheng, T. H. Li, Y. C. Lin, L. Y. Yang, C. P. Lin, C. S. Hou, H. C. Lin, J. L. Yang, K. F. Yu, M. J. Chen, T. H. Hsieh, Y. C. Peng, C. H. Chou, C. J. Lee, C. W. Huang, C. Y. Lu, F. K. Yang, H. K. Chen, L. W. Weng, P. C. Yen, S. H. Wang, S. W. Chang, S. W. Chuang, and T. C. Ga. 2010. High performance 22/20nm FinFET CMOS devices with advanced high-K/metal gate scheme. In Proc. IEEE Int. Electron Devices Meeting. 27.1.1-27.1.4.

S. Xiong and J. Bokor. 2003. Sensitivity of double-gate and FinFET devices to process variations. IEEE Trans. Electron Devices 50, 11 (Nov. 2003), 2255-2261.

T. Yamashita, V. S. Basker, T. Standaert, C. C. Yeh, T. Yamamoto, K. Maitra, C. H. Lin, J. Faltermeier, S. Kanakasabapathy, M. Wang, H. Sunamura, H. Jagannathan, A. Reznicek, S. Schmitz, A. Inada, J. Wang, H. Adhikari, N. Berliner, K. L. Lee, P. Kulkarni, Y. Zhu, A. Kumar, A. Bryant, S. Wu, T. Kanarsky, J. Cho, E. Mclellan, S. J. Holmes, R. C. Johnson, T. Levin, J. Demarest, J. Li, P. Oldiges, J. Arnold, M. Colburn, M. Hane, D. Mcherron, V. K. Paruchuri, B. Doris, R. J. Miller, H. Bu, M. Khare, J. ONeill, and E. Leobandung. 2011. Sub-25nm FinFET with advanced fin formation and short channel effect engineering. In Proc. Symp. VLSI Technol. 14-15.

Y. Yang and N. K. Jha. 2014. FinPrin: FinFET logic circuit analysis and optimization under PVT variations. IEEE Trans. VLSI Syst. 22, 12 (Dec. 2014), 2462-2475.

R. Zhang, M. R. Stan, and K. Skadron. 2015. HotSpot 6.0: Validation, acceleration and extension. University of Virginia Tech. Report CS-20 (2015), 15-04.

Received September 2016; revised March 2017; accepted June 2017 